\title{
A density-temperature description of the outer electron radiation belt during geomagnetic storms
}

\author{
Michael H. Denton, ${ }^{1}$ Joseph E. Borovsky, ${ }^{2}$ and Thomas E. Cayton ${ }^{2}$ \\ Received 20 February 2009; revised 9 July 2009; accepted 26 August 2009; published 21 January 2010.
}

[1] Bi-Maxwellian fits are made to energetic-electron flux measurements from seven satellites in geosynchronous orbit, yielding a number density (n) and temperature (T) description of the outer electron radiation belt. For 54.5 spacecraft years of measurements the median value of $\mathrm{n}$ is $3.7 \times 10^{-4} \mathrm{~cm}^{-3}$, and the median value of T is $148 \mathrm{keV}$. General statistical properties of $\mathrm{n}$, $\mathrm{T}$, and the $1.1-1.5 \mathrm{MeV}$ flux $\mathrm{F}$ are investigated, including localtime and solar-cycle dependencies. Using superposed-epoch analysis where the zero epoch is convection onset, the evolution of the outer electron radiation belt through high-speed-stream-driven storms is investigated. The number-density decay during the calm before the storm, relativistic-electron dropouts and recoveries, and the heating of the outer electron radiation belt during storms are analyzed. Using four different "triggers" (sudden storm commencement (SSC), southward interplanetary magnetic field (IMF) portions of coronal mass ejection (CME) sheaths, southward-IMF portions of magnetic clouds, and minimum Dst) a selection of CME-driven storms are analyzed with superposed-epoch techniques. For CME-driven storms, only a very modest density decay prior to storm onset is found. In addition, the compression of the outer electron radiation belt at the time of SSC is analyzed, the number-density increase and temperature decrease during storm main phase are characterized, and the increase in density and temperature during storm recovery phase is determined. During the different phases of storms, changes in the flux are sometimes in response to changes in the temperature, sometimes to changes in the number density, and sometimes to changes in both. Differences are found between the density-temperature and flux descriptions, and it is concluded that more information is available using the density-temperature description.

Citation: Denton, M. H., J. E. Borovsky, and T. E. Cayton (2010), A density-temperature description of the outer electron radiation belt during geomagnetic storms, J. Geophys. Res., 115, A01208, doi:10.1029/2009JA014183.

\section{Introduction}

[2] A major issue in magnetospheric physics is to understand the evolution of the radiation belts. The evolution of the outer electron radiation belt involves the addition and loss of electrons, the radial transport of electrons, and the heating and cooling of electrons. The density-temperature measurements presented here provide a new look at the behavior of the outer electron radiation belt during two types of geomagnetic storms.

[3] Relativistic electrons are harmful to satellite systems and instrumentation [e.g., Wrenn, 2009], present significant dangers to cosmonauts passing through the region, and have also been implicated as playing a limited role in the morphology of stratospheric ozone [e.g., Callis et al., 1991; Pesnell et al., 2000; Roldugin et al., 2000; Sinnhuber et al., 2006; Seppälä et al., 2007]. In general, differential

\footnotetext{
${ }^{1}$ Department of Communication Systems, Lancaster University, Lancaster, UK.

${ }^{2}$ Space Science and Applications, Los Alamos National Laboratory, Los Alamos, New Mexico, USA.

Copyright 2010 by the American Geophysical Union. 0148-0227/10/2009JA014183\$09.00
}

fluxes are used to describe the properties of the electron radiation belt and are used in studies to determine acceleration and loss mechanisms [e.g., Paulikas and Blake, 1979; Nagai, 1982; Friedel et al., 2002; Reeves et al., 2003; Mann et al., 2004; Li et al., 2005; Liemohn and Chan, 2007; Onsager et al., 2007; Millan and Thorne, 2007; Summers et al., 2007a, 2007b; Baker and Kanekal, 2008; Hudson et al., 2008; Borovsky and Denton, 2009a]. However, fluxes are somewhat ambiguous in that increases in the flux can be caused by increases in the number of electrons or by a heating of the electrons, and likewise decreases in the flux can be caused by decreases in the number of electrons or by a cooling of the electrons.

[4] A density and temperature description of the electron radiation belt provides substantial advantages over the flux description: changes in the number of electrons and changes in the temperature of the electrons are separated in the measurements. With slight heating or cooling of the electron population the relativistic-electron fluxes can change by orders of magnitude. A flux description leads one to look for a particle-acceleration mechanism that can boost energies by orders of magnitude whereas a density-temperature description leads one to look for a heating mechanism that can 
modestly heat the electrons [cf. Borovsky et al., 1998]. Hence, a density-temperature description provides a simplified and more manageable description of the evolution of the radiation belts, with the potential to reveal physical phenomena not readily accessible with a flux description.

[5] The density-temperature description of the outer electron radiation belt that is used in this study was developed by Cayton et al. [1989]. In Cayton et al.'s [1989] study, relativistic bi-Maxwellian fits of the multisatellite CPA (charged particle analyzer) measurements were carried out to investigate the effect of magnetospheric substorms on the outer electron radiation belt. The study revealed that substorms result in decreases in the number density of the outer electron radiation belt at geosynchronous orbit. That study also found 27-day periodicities in the number density of the outer electron radiation belt. Other studies have since utilized this description of the electron radiation belt. Borovsky et al. [1998] used Cayton's relativistic bi-Maxwellian fits to multisatellite SOPA (synchronous orbit particle analyzer) measurements of the outer electron radiation belt at geosynchronous orbit in order to study the evolution of the outer electron radiation belt during storms. Borovsky et al. [1998] observed that after the outer electron radiation belt was emptied of electrons at storm onset, the number density of electrons rapidly returned to a level that remained quasiconstant during the storm itself, and that slow increases in the relativistic-electron flux during the storms were associated with a slow and modest heating of the outer electron radiation belt at constant density. Borovsky and Steinberg [2006] used Cayton's relativistic bi-Maxwellian fits to the multisatellite SOPA measurements to examine the effect of the outer plasmasphere on the outer electron radiation belt during calm periods before storms. They found that the number density of the outer electron radiation belt at geosynchronous orbit decays prior to storm onset and that the decay is stronger when the outer plasmasphere is present in the magnetosphere than when it is absent. Borovsky and Denton [2009b] followed up that study to quantify the number-density decay rates of the outer electron radiation belt number density during calms before storms.

[6] The current study presents a density-temperature overview of the evolution of the outer electron radiation belt at geosynchronous orbit during geomagnetic storms driven by high-speed solar wind streams (HSSs) and geomagnetic storms driven by coronal mass ejections (CMEs). Previous work has revealed that the two types of solar wind have distinct physical consequences for the inner magnetosphere [e.g., Lindsay et al., 1995; Wilken et al., 1999; Denton et al., 2006; Borovsky and Denton, 2006a; Lavraud et al., 2006; Georgieva et al., 2006; Turner et al., 2006; Longden et al., 2008; Sandanger et al., 2009]. In the current study, more distinctions between the two types of solar wind driver are quantified in detail in terms of the electron temperature and number-density response of the outer radiation belt.

[7] This manuscript is organized as follows. In section 2 the relativistic bi-Maxwellian fits to energetic electrons are overviewed and the data set that will be used are described. In section 3 the evolution of the outer electron radiation belt at geosynchronous orbit during high-speed-stream-driven storms is analyzed using superposed-epoch techniques. In section 4 the evolution of the outer electron radiation belt at geosynchronous orbit during coronal-mass-ejection-driven storms is examined using superposed-epoch data analysis with four separate "triggers" to discern the evolution during different phases of the storms. The findings of this study are summarized in section 5. Ideas for future work are discussed in section 6 .

\section{Data Analysis: Relativistic Bi-Maxwellian Fits}

[8] The SOPA instruments [Belian et al., 1992; Cayton and Belian, 2007] measure spin-averaged energy-resolved fluxes of energetic electrons in the energy range $\sim 50 \mathrm{keV}$ to $>2 \mathrm{MeV}$ at a cadence of $10 \mathrm{~s}$. Cayton et al. [1989] found that the energetic-electron energy distribution at geosynchronous orbit could be well described by relativistic bi-Maxwellians. (Note that Pierrard and Lemaire [1996] found that electronradiation belt energy spectra away from geosynchronous orbit were also well described by relativistic bi-Maxwellians.) For the present data analysis the electron measurements at geosynchronous orbit measured by SOPA instruments [Belian et al., 1992] on seven Los Alamos National Laboratory (LANL) satellites $(046,095,080,084,97 \mathrm{~A}, 01 \mathrm{~A}$, and 02A) in the 18 years from 1990 through 2007 are fitted with relativistic bi-Maxwellian distributions using a method refined from the method of Cayton et al. [1989]. The current method models the counting rates for each electron energy channel as linear combinations of two Maxwellian components plus a nonelectron "background" contribution. Minimizing the squared deviations between the observed and model counting rates summed over 10 electron channels yields the best fitting two Maxwellian spectra (see also Cayton and Belian [2007] for full details). A scatterplot of the number densities and temperatures of the two components of the bi-Maxwellian fits appears in Figure 1. The points are half-hour median values of the fit parameters for the spacecraft LANL-01a for the seven years 2001-2007. The energetic electrons at geosynchronous orbit divide into a "hard" component with a number density of $\sim 5 \times 10^{-4} \mathrm{~cm}^{-3}$ and a temperature of $\sim 150 \mathrm{keV}$ (red points in Figure 1) and a "soft" component with a number density of $\sim 1 \times 10^{-2} \mathrm{~cm}^{-3}$ and a temperature of $\sim 30 \mathrm{keV}$ (green points in Figure 1). The soft component is the suprathermal tail of the electron plasma sheet, which is associated with substorm energeticelectron injections [e.g., Cayton et al., 1989; Birn et al., 1998]. The hard component is the outer electron radiation belt. Note in Figure 1 that the temperature of the outer electron radiation belt (red points) varies by factors of $\sim 2$ whereas the number density varies by more than an order of magnitude.

[9] In Figure 2 the number density $\mathrm{n}$, temperature $\mathrm{T}$, and pressure (energy density) $\mathrm{P}=\mathrm{nk}_{\mathrm{B}} \mathrm{T}$ of the outer electron radiation belt at geosynchronous orbit are plotted along with the multisatellite average of relativistic-electron flux in the 1.1-1.5 MeV energy band [Borovsky and Denton, 2009a]. For Figures $2 \mathrm{a}-2 \mathrm{c}$ the binning is of 955,527 half-hour medians comprising 54.5 satellite years of data taken between 1990 and 2007. Figure 2a shows the occurrence distribution of $\log _{10}(n)$. The distribution of $\log _{10}(n)$ has a modest skewness (i.e., it is slightly asymmetric) and a modest kurtosis (i.e., it is somewhat peaked): the distribution of $\mathrm{n}$ itself has a large skewness and an extraordinarily large kurtosis. The mean value of $\mathrm{n}$ is $4.2 \times 10^{-4} \mathrm{~cm}^{-3}$ and 


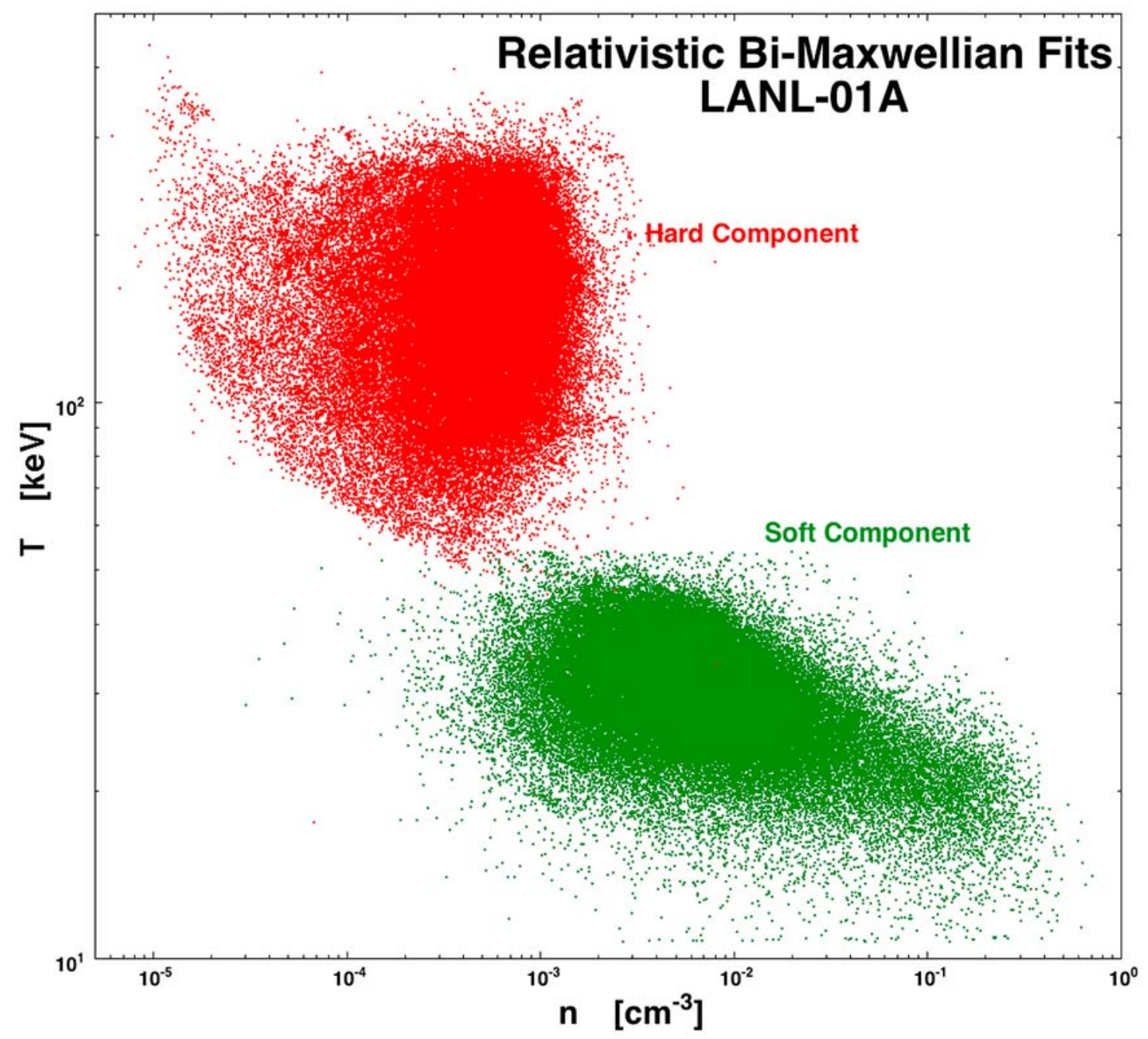

Figure 1. A scatterplot containing half-hour median values of number density and temperature for the spacecraft LANL-01a for the seven years 2001-2007, calculated from two-component relativistic Maxwellian fits. The energetic electrons at geosynchronous orbit divide into a "hard" component with a number density of $\sim 5 \times 10^{-4} \mathrm{~cm}^{-3}$ and a temperature of $\sim 150 \mathrm{keV}$ (red points) and a "soft" component with a number density of $\sim 1 \times 10^{-2} \mathrm{~cm}^{-3}$ and a temperature of $\sim 30 \mathrm{keV}$ (green points).

the median value of $\mathrm{n}$ is $3.7 \times 10^{-4} \mathrm{~cm}^{-3}$. The number density of the outer electron radiation belt at geosynchronous orbit is about $10^{-3}$ times as small as the number density of hot plasma at geosynchronous orbit [cf. Denton et $a l ., 2005]$. Figure $2 \mathrm{~b}$ shows the occurrence distribution of the temperature $\mathrm{T}$ of the electron radiation belt. The distribution of $\mathrm{T}$ has a slight skewness and a slight kurtosis. The mean value of $\mathrm{T}$ is $142 \mathrm{keV}$ and the median value of $\mathrm{T}$ is $148 \mathrm{keV}$. Note that the relative variation of $\mathrm{T}$ (standard deviation divided by mean value) is about $30 \%$; the relative variance of $n$ is about $70 \%$. In Figure $2 c$ the occurrence distribution of the pressure $\mathrm{P}=\mathrm{nk}_{\mathrm{B}} \mathrm{T}$ of the outer electron radiation belt at geosynchronous orbit is shown. The distribution of the pressure itself has a large skewness and a very large kurtosis. The mean value of $\mathrm{P}$ is $0.0104 \mathrm{nPa}$ and the median value is $0.0083 \mathrm{nPa}$. In Figure $2 \mathrm{~d}$ the differential flux $\mathrm{F}$ of relativistic electrons in the $1.1-1.5 \mathrm{MeV}$ energy band are binned. The binning is of 876,391 half-hour medians comprising 50.0 satellite years of data taken between 1989 and 2006. These data were originally utilized in the study of Borovsky and Denton [2009a]. Figure 2d shows the occurrence distribution of $\log _{10}(F)$. Comparison between Figures $2 a-2 c$ and Figure $2 d$ highlights the difference between the density/temperature distributions and the flux distribution. The distribution of $\log _{10}(\mathrm{~F})$ has a modest skewness and a modest kurtosis: the distribution of $F$ itself has a large skewness and an extraordinarily large kurtosis. The mean value of $\mathrm{F}$ is $13.0 \mathrm{~cm}^{-2} \mathrm{~s}^{-1} \mathrm{ster}^{-1} \mathrm{keV}^{-1}$ and the median value is $4.7 \mathrm{~cm}^{-2} \mathrm{~s}^{-1} \mathrm{ster}^{-1} \mathrm{keV}^{-1}$. By comparing Figures $2 \mathrm{a}$ and $2 \mathrm{~d}$, it is clear that the occurrence distribution of $\log _{10}(\mathrm{n})$ has a width of about 1.5 (which is 1.5 orders of magnitude of variation of $n$ ), while the occurrence distribution of $\log _{10}(\mathrm{~F})$ has a width of about 2.5 (which is 2.5 orders of magnitude of variation of $F$ ). The relative variation of $F$ (standard deviation divided by mean value) is about $180 \%$ which is much larger than the relative variation of $\mathrm{n}$ or $\mathrm{T}$ indicating that the flux distribution is more variable that either the temperature or density distribution. 

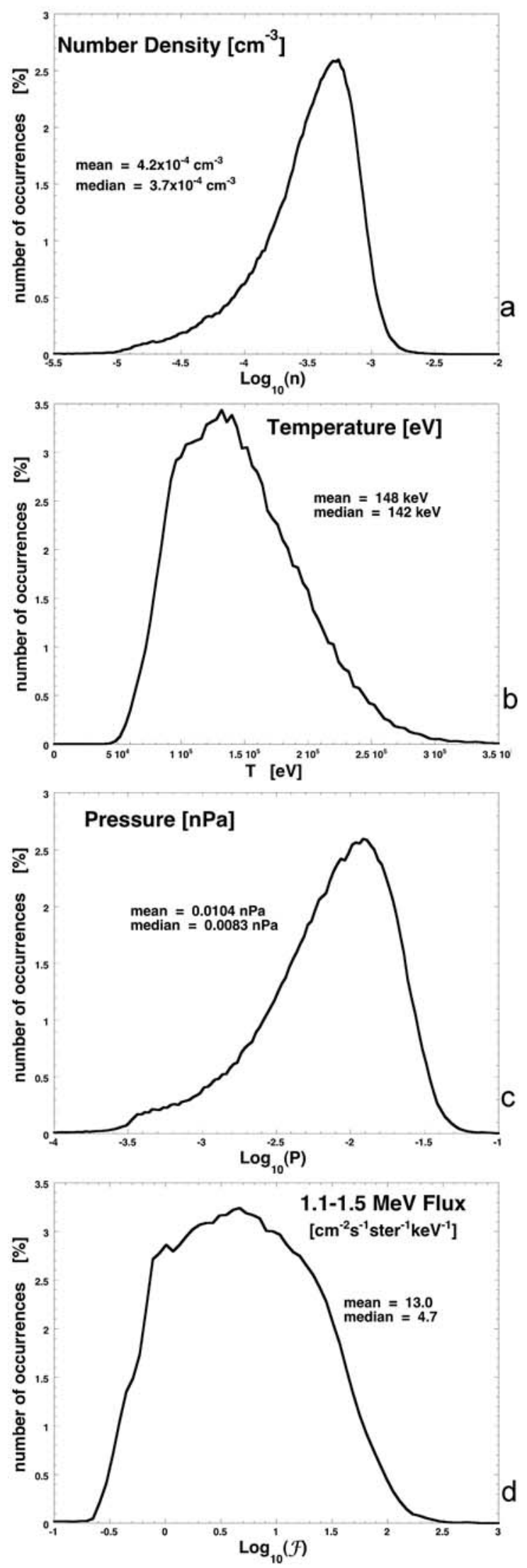

[10] As measured at geosynchronous orbit, there are local-time dependencies in the number density n, temperature $\mathrm{T}$, and electron flux $\mathrm{F}(1.1-1.5 \mathrm{MeV})$ of the outer electron radiation belt. These local-time dependencies are shown in Figure 3. The gray points are the half-hour measurements (with every 20th point plotted) and the red points are 3000-point running logarithmic averages of the gray points. Note that the number density (Figure 3a) of the outer electron radiation belt at geosynchronous orbit has a weak local-time dependence with a maximum in the prenoon sector between 0900 and $1000 \mathrm{LT}$; the temperature (Figure 3b) has a similar but weaker local-time dependence with a maximum between 1100 and 1200 LT. The relativisticelectron flux $\mathrm{F}$ in the $1.1-1.5 \mathrm{MeV}$ energy band at geosynchronous orbit is also plotted as a function of local time (Figure 3c). The flux also shows a similar local-time dependence as the number density $\mathrm{n}$ and the temperature $\mathrm{T}$ of the electrons with a maximum between 1100 and 1200 LT. In general, the equatorial magnetic field strength at geosynchronous orbit is weaker on the nightside than it is on the dayside. This can be seen in Figure 3d, where half-houraveraged values of the magnetic field strength as measured by the GOES 10 spacecraft in geosynchronous orbit in the years 1999 through 2003 are plotted (the maximum field strength occurs between 1100 and 1200 LT). To ensure that the GOES spacecraft was not in the magnetosheath owing to magnetopause crossings, GOES magnetometer measurements were only used if the magnetic field clock angle measured by GOES was within $20^{\circ}$ of the dipole direction; magnetopause crossings at geosynchronous orbit almost always occur under southward IMF [e.g., Rufenach et al., 1989], which would put the clock angle in the magnetosheath at $90^{\circ}$ or more from the dipole direction. The red curve is a 300-point running average of the gray points. Energetic electrons drift in a manner preserving their adiabatic invariants; to preserve their first adiabatic invariant they tend to drift on orbits with constant magnetic field strength at the equator. Hence, they move further out on the dayside (where the field is strong) and closer in on the nightside (where the field is weak) [e.g., Roederer, 1967]. At geosynchronous orbit on the nightside a spacecraft is in the more outer portions of the radiation belt than is a spacecraft on the dayside. Since the radiation belts are of lower density and cooler further out, a nightside spacecraft sees a lower density and a lower temperature than does a dayside spacecraft (cf. Figures $3 a$ and $3 b$ ). The flux (Figure 3c) follows the density-temperature trend. Note that some recent data-analysis efforts [e.g., Onsager et al., 2004; Green and Kivelson, 2004; Chen et al., 2005] have used magnetic field models to transform measurements taken at geosynchronous orbit $\left(6.6 \mathrm{R}_{\mathrm{e}}\right)$ to coordinates reflecting the noncircular orbits of the radiation-belt electrons in order to remove adiabatic

Figure 2. The (a) number density, (b) temperature, (c) pressure, and (d) relativistic-electron flux $(1.1-1.5 \mathrm{MeV})$ of the outer radiation belt at geosynchronous orbit, binned to produce occurrence distributions (1990-2007). For the number density, temperature, and pressure the base-10 logarithms are binned $(955,527$ half-hour measurements from seven satellites), while for the flux, the half-hour multisatellite averages are binned (278,471 data points). 

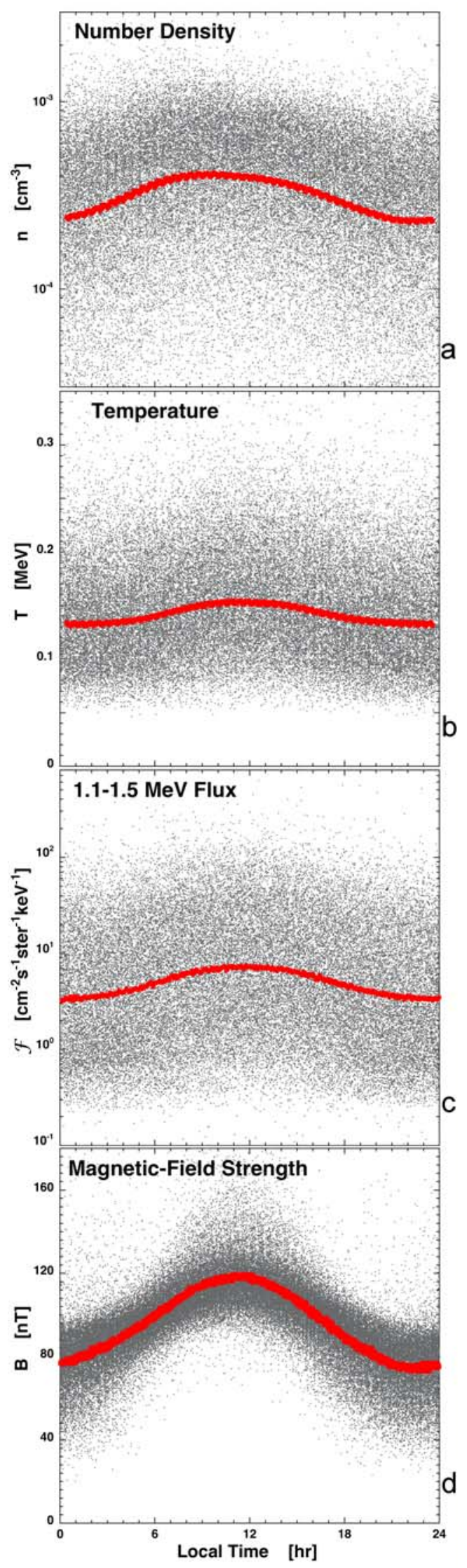

"Dst effects" [Dessler and Karplus, 1961] and pressureinduced changes [Zaharia et al., 2005]. Such model-dependent techniques are not utilized in the present investigation: here, the radiation environment at geosynchronous orbit itself is studied.

[11] In Figure 4 the density $n$ (Figure 4a), temperature $T$ (Figure $4 \mathrm{~b}$ ), pressure $\mathrm{P}=\mathrm{nk}_{\mathrm{B}} \mathrm{T}$ (Figure $4 \mathrm{c}$ ), and $1.1-1.5 \mathrm{MeV}$ flux $F$ (Figure 4d) of the outer electron radiation belt at geosynchronous orbit are plotted as a function of time for 18 years. The international sunspot number (ISN) is also plotted (Figure 4e). In Figures $4 a-4 c$ the black points are 5000-point logarithmic averages of the 955,527 half-hour measurements. Depending on the number of geosynchronous satellites in operation at the time, the 5000-point averaging represents about a 1 month running average. In Figure $4 \mathrm{~d}$ the black points are 1440-point logarithmic averages (a 30 day running average) of the 278,471 halfhour multisatellite averages of the fluxes, F. These fluxes are the multisatellite averaged fluxes calculated from SOPA instruments on all available spacecraft and previously used in Borovsky and Denton's [2009a] study. As can be seen, during years with substantial high-speed-stream activity, such as 1993-1994, 2003, and 2005 (in declining phases of the solar cycle), the number density $n$ of the outer electron radiation belt at geosynchronous orbit tends to be high. Some years around solar minimum (e.g., 1995 and 2007), the outer electron radiation belt tends to have a very low density. The pressure (energy content) of the outer electron radiation belt (Figure 4c) tends to reflect these number-density trends. The solar-cycle temperature variations are less than the variations in number density, with the temperature of the outer electron radiation belt tending to be slightly higher than average during the declining phase of the solar cycle. The monthly averaged relativistic-electron flux $\mathrm{F}$ at geosynchronous orbit also tends to reflect the trend in the number density of the outer electron radiation belt: the flux is higher during the declining phase of the solar cycle than it is during the other phases.

\section{Evolution of the Outer Electron Radiation Belt During High-Speed Solar Wind Streams}

[12] The arrival of high-speed solar wind streams (HSSs) at the Earth's magnetosphere initiates numerous physical processes, including (1) a sudden onset of magnetospheric convection (e.g., McPherron and Weygand [2006] and

Figure 3. (a, b) For 955,527 half-hour measurements (1990-2007), the number density and temperature of the outer electron radiation belt at geosynchronous orbit are plotted as a function of the local time at which the measurement was made. (c) For 876,391 half-hour measurements (1999-2003), the $1.1-1.5 \mathrm{MeV}$ electron flux is plotted as a function of the local time at which the measurement was made. (d) For 82,579 half-hour measurements (1989-2006) from the GOES 10 spacecraft, the magnetic field strength at geosynchronous orbit is plotted as a function of the local time at which the measurement was made. The gray points are the individual measurements, and the red points are 3000 -point averages of the gray points. 

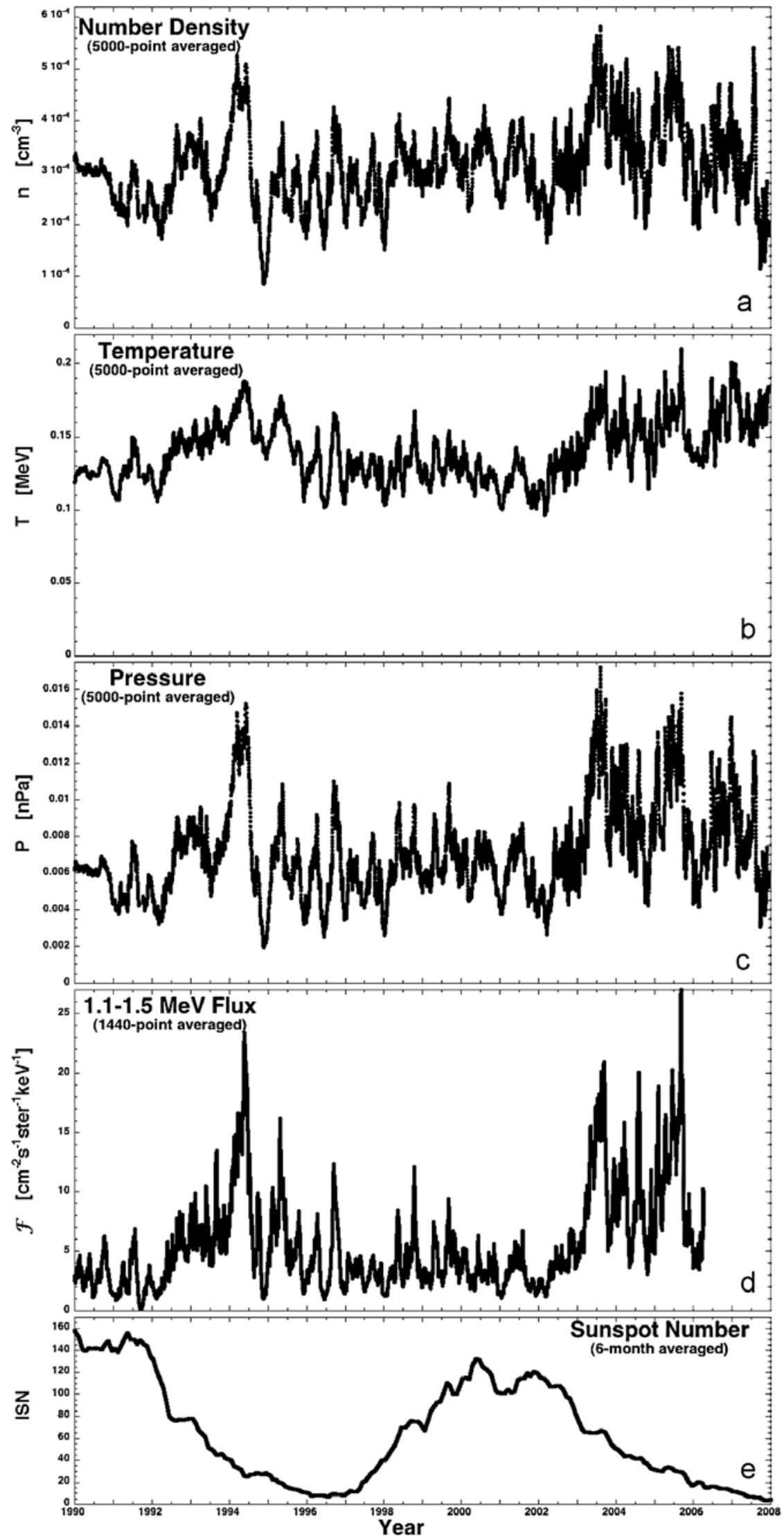

Figure 4 
compare Figure 5), (2) enhanced density, heating, and transport in the plasma sheet [e.g., Borovsky et al., 1998; Lavraud et al., 2006; Denton and Borovsky, 2008], (3) enhanced particle precipitation [Longden et al., 2008], (4) flux dropouts/recovery of outer radiation belt electrons [e.g., Paulikas and Blake, 1979; O'Brien et al., 2001; Borovsky and Denton, 2009a], (5) the formation of longlived plasmaspheric drainage plumes [Borovsky and Denton, 2006b, 2008], and (6) an increase in ionospheric heating and the occurrence of $F$ region storms [Denton et al., 2009b; Sojka et al., 2009]. Many of these processes are interlinked [e.g., Tsurutani et al., 2006; Kavanagh and Denton, 2007; Denton et al., 2009a]. For example, the onset of convection (process 1) causes plasma from the outer plasmasphere to be stripped away into a plasmaspheric plume (process 5), which is implicated in the loss of relativistic electrons from the outer radiation belt (process 4) [Borovsky and Denton, 2009a].

[13] To study the behavior of the outer electron radiation belt during high-speed-stream-driven storms we perform a superposed-epoch analysis of 124 HSS storms that occurred between 1993 and 2006. Previously, these events have been used successfully in studies of the magnetospheric response to HSSs [Borovsky and Denton, 2008, 2009a, 2009b; Denton and Borovsky, 2008, 2009; Denton et al., 2009b]. The list of HSSs was constructed on the basis of an initial list of CIR stream interfaces for 1993-1996 provided by R. McPherron (private communication, 2005): the HSS is the extended period of fast solar wind which follows the CIR. More events were added to the initial list by searching solar wind data for years beyond 1996 and identifying typical signatures of HSSs (e.g., east-west flow deflection followed by sustained elevated solar wind speed). The final list of HSS storms between 1993 and 2006 includes an "onset time," which is the time of storm convection onset. Convection onsets are initially detected by a strong rise in the Kp index [cf. Elphic et al., 1999; Thomsen, 2004], with the final onset time calculated to $\sim 30$ min time resolution using changes in the Midnight Boundary Index (MBI) [Madden and Gussenhoven, 1990]. It should be noted that events with no discernable increase in convection following a HSS identification in the solar wind are not included in the study (see Denton et al. [2006] and Denton and Borovsky [2008] for full details). This event selection likely corresponds to events that are Russell-McPherron effective [Russell and McPherron, 1973; McPherron et al., 2009].

[14] Figure 5 contains plots of a selection of superposed solar wind, radiation belt, and geomagnetic parameters for 35 days prior to storm onset (zero epoch) and 35 days after storm onset; that is, it encompasses more than one solar rotation period before and after the arrival of each HSS at the magnetosphere. In Figure 5a the superposition of the solar wind speed (V-SW) is show for the 124 high-speedstream-driven storms. The dark blue curve in each plot of Figure 5 is the mean value of the data superposition. The speed is obtained from the high-resolution OMNI2 database [King and Papitashvili, 2005]. The solar wind speed plot indicates that prior to onset the speed reaches a local minimum before increasing sharply (see Borovsky and Steinberg [2006] for a discussion of the "calm before the storm"). At storm onset the speed is increasing and the speed remains at an elevated level for a number of days. Typically storm onset occurs during the passage of the corotating interaction region (CIR) upstream of the highspeed wind [Denton and Borovsky, 2009]. The east-west component of the solar wind (VY-SW) shown in Figure $5 \mathrm{~b}$ indicates the rapid negative-then-positive flow deflection of the CIR: the onset of the HSS-driven storm occurs within the CIR [see also Denton and Borovsky, 2008, Figure 1]. The superposition of the north-south (GSM) component of the interplanetary field $\left(\mathrm{B}_{\mathrm{Z}}-\mathrm{GSM}\right)$ for the 124 high-speedstream-driven storms is shown in Figure 5c. The superposition of $\mathrm{B}_{\mathrm{Z}}$ indicates that on average there is a strong southward turning of the IMF close to the storm onset, although some of the events do have northward $\mathrm{B}_{\mathrm{Z}}$ at this time. In Figure $5 \mathrm{~d}$ the superposition of the solar wind number density (N-SW) is plotted for the 124 high-speedstream-driven storms. As can be seen, there is a peak in the superposed solar wind density that is roughly concurrent with the strong southward $\mathrm{B}_{\mathrm{Z}}$ field. The peak in density is the result of the compression of the solar wind within the CIR and the peak in solar wind density produces a superdense plasma sheet in the magnetosphere (when the solar wind plasma is captured and heated) [Borovsky et al., 1997; Denton and Borovsky, 2008, 2009]. Following this peak, the solar wind density falls to a level below its prestorm average. The geomagnetic reaction of the magnetosphere during the high-speed-stream-driven storms can be gauged in Figures 5e and 5f, where superpositions of the Dst index and the $K p$ index are shown for the 124 events. At time $\mathrm{t}=0$ there is a sharp increase in the value of $K p$ (storm onset) and $K p$ persists at elevated levels for several days after storm onset. Note in Figure $5 \mathrm{f}$ that prior to the increase in $K p$ there is a distinct period of lower-than-normal $K p$ in the data superposition: this is the "calm before the storm" [Clilverd et al., 1993; Borovsky and Steinberg, 2006; Borovsky and Denton, 2009b]. Note also in Figure 5f the 27-day periodicity that is seen in the increase of $K p$ associated with the 27-day recurrence of the high-speed streams. In Figure 5e the superposition of the Dst index is plotted for the 124 high-speed-stream-driven storms. One feature to note is the limited decrease in Dst near zero epoch. On average, these high-speed-stream-driven storm events have a minimum $D s t$ of around -30 . Indeed, it is instructive to note that by simply relying on Dst as a proxy for "storm strength," the majority of the events used in this study would likely be neglected [cf. Denton et al., 2006; Borovsky and Denton, 2006a; Georgieva et al., 2006].

[15] Figure 6 contains epoch time versus local time plots of the superposed relativistic-electron number density (n),

Figure 4. Plots of the (a) density, (b) temperature, (c) pressure, and (d) flux (1.1-1.5 MeV), of the outer electron radiation belt at geosynchronous orbit as a function of time for 18 years. (e) Plot of the international sunspot number (ISN). In Figures $4 \mathrm{a}-4 \mathrm{c}$ the black points are 5000-point logarithmic averages of the 955,527 half-hour measurements from seven satellites (1990-2007 inclusive). In Figure 4d the black points are 1440-point logarithmic averages of 278,471 half-hour multisatellite averages (1990-2006). 

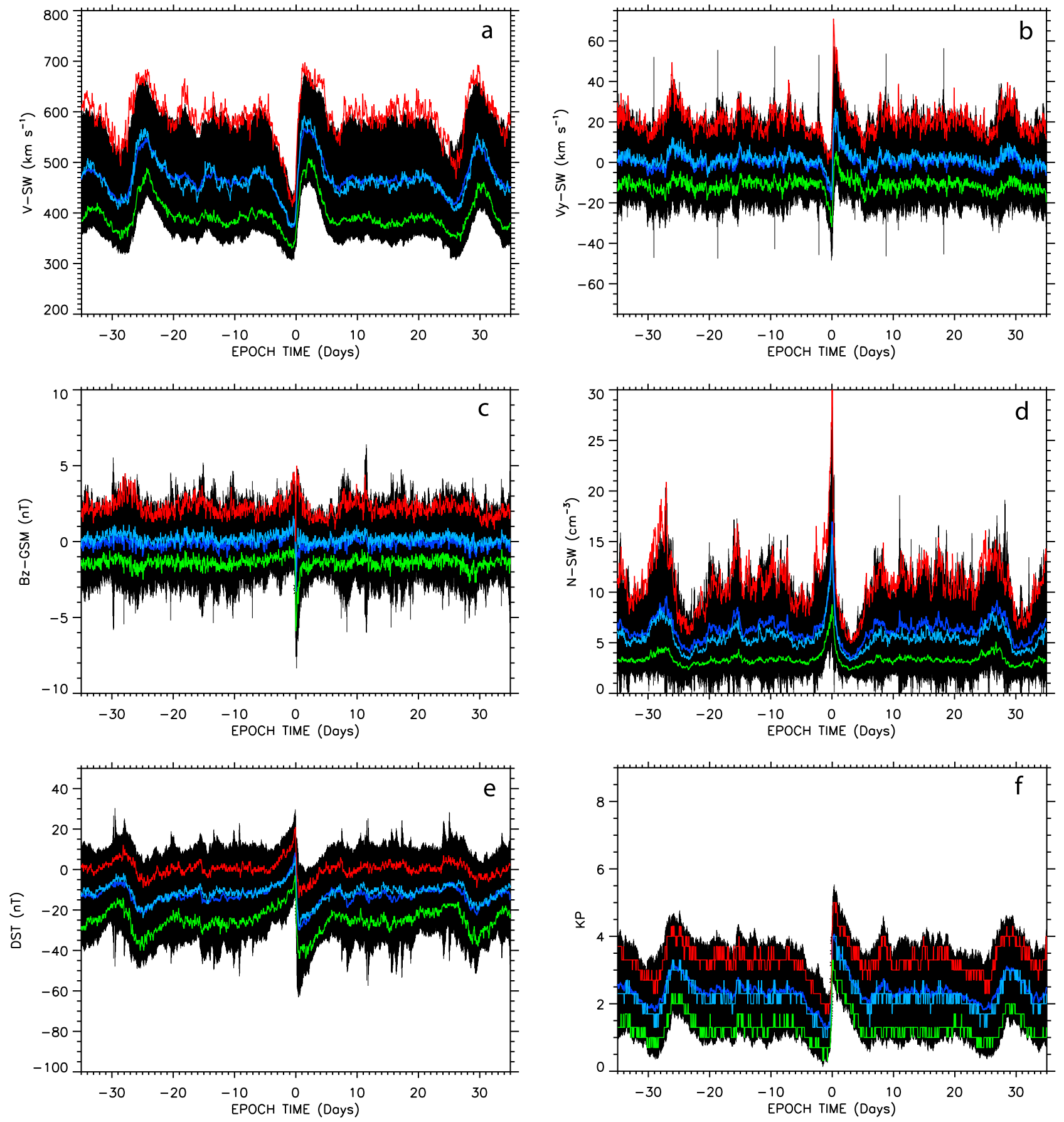

Figure 5. $(\mathrm{a}-\mathrm{f})$ Selected solar wind parameters as a function of epoch time for 124 HSSs between 1993 and 2006. The upper quartiles on the measurements are shown in red, while the lower quartiles are shown in green. The mean value of each parameter is plotted in dark blue, while the median is plotted in light blue. The gray shaded regions indicate values within one standard deviation of the mean.

temperature $(\mathrm{T})$, pressure $\left(\mathrm{P}=\mathrm{nk}_{\mathrm{B}} \mathrm{T}\right)$, and relativisticelectron flux $\mathrm{F}(1.1-1.5 \mathrm{MeV})$ for $124 \mathrm{HSSs}$ between 1993 and 2006. The zero epoch for the superposition is again the storm onset of magnetospheric convection. The fluxes $\mathrm{F}$ used here are the multisatellite averaged fluxes calculated from SOPA instruments on all available spacecraft and previously used in Borovsky and Denton's [2009a] study. The plots extend from 35 days prior to onset to 35 days after onset. Figure 6 contains a broad overview of the relativistic-electron behavior in the outer radiation belt at geosynchronous orbit during HSSs, and several features are clearly apparent. First, Figure 6 shows that the density, temperature, and pressure of the outer electron radiation belt all decrease at close to zero epoch. Second, Figure 6 shows that the number density, temperature, and pressure of the outer radiation belt all increase after storm onset, as does the relativistic-electron flux. Following the decrease in density and temperature around zero epoch, all three parameters are 


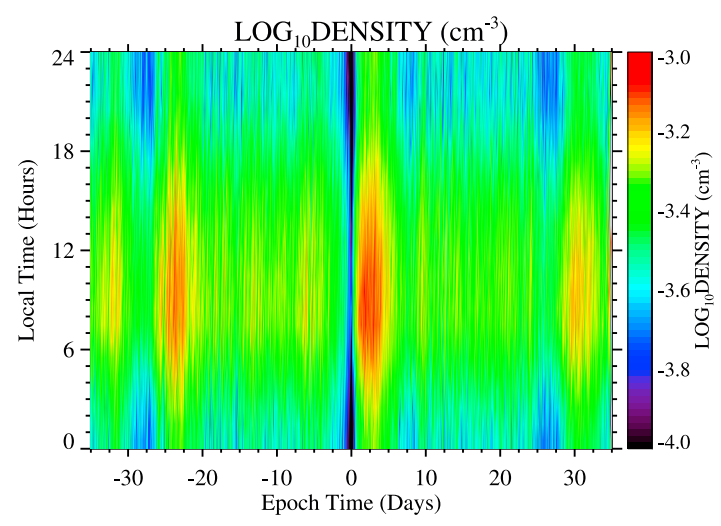

then elevated for a period of $\sim 4$ days duration. This is consistent with the duration of the high-speed solar wind (Figure 5a), and associated elevated levels of convection (Figure 5f). Third, Figure 6 shows that there is a pronounced quasi-27-day periodicity in all of the outer-electron-radiationbelt parameters owing to the presence of recurrent high-speed solar wind structures (cf. Figure 5). The local-time dependence of the number density, temperature, pressure (energy density), and the relativistic-electron flux in Figure 6 follows the trends described in section 2. The outer electron radiation belt at geosynchronous orbit is more intense on the dayside than on the nightside owing to the weaker magnetic field on the nightside (compare Figure 3).

[16] The detailed behavior of electrons within the outer radiation belt during a HSS-driven storm can better be grasped by examination of Figure 7. Here, the zero epoch is the onset of storm levels of convection and time extends from 5 days prior to storm onset to 5 days after storm onset. In Figures 7a, 7c, 7e, and $7 \mathrm{~g}$ the superposed number density, temperature, pressure, and relativistic-electron flux (1.1$1.5 \mathrm{MeV}$ ) of the outer electron radiation belt at geosynchronous orbit are plotted in local time versus epoch time. In Figures $7 \mathrm{~b}, 7 \mathrm{~d}, 7 \mathrm{f}$, and $7 \mathrm{~h}, 3 \mathrm{~h}$ wide local-time cuts (2300-0100 LT, 0500-0700 LT, 1100-1300 LT, and $1700-1900$ LT) of the number density, temperature, pressure, and flux are plotted versus time. Each curve plotted in Figures $7 \mathrm{~b}, 7 \mathrm{~d}, 7 \mathrm{f}$, and $7 \mathrm{~h}$ is smoothed with a $10 \mathrm{~h}$ boxcar average in time. Four features seen in Figure 7 are discussed in the following four paragraphs and are summarized in Table 1.

[17] First, in the few days prior to storm onset, there is a slow decay in the number density of the outer electron radiation belt at geosynchronous orbit while the temperature remains constant. This is best observed in the line plots of number density and temperature shown in Figure 7: the number density decreases at all local times while the temperature is approximately constant at all local times. The density begins to decrease about four days prior to storm onset. Owing to this density decay, the pressure also decreases with time in the days prior to storm onset (Figures $7 \mathrm{e}$ and $7 \mathrm{f}$ ) and the relativistic-electron flux also decreases with time (Figures $7 \mathrm{~g}$ and $7 \mathrm{~h}$ ). These trends are recorded in Table 1. Note in Figure 7 that the logarithmic decrease in the relativistic-electron flux in the days before storm onset is only about half the logarithmic decrease in the number density. In the days prior to the onset of HSSdriven storms, a geomagnetic calm tends to occur owing to a Russell-McPherron effect [Borovsky and Steinberg, 2006]. The decay of the number density of the outer electron radiation belt during these calms prior to storms is attributable to the refilling of the outer plasmasphere. This refilling in turn leads to a pitch angle scattering of the radiation belt electrons into the atmospheric loss cone [e.g., Meredith et al., 2004] and eventual precipitation into

Figure 6. Superposed outer electron radiation belt number density, temperature, pressure, and flux, calculated using a superposed-epoch analysis of 124 high-speed solar wind streams between 1993 and 2006. Averaged values of these parameters at $1 \mathrm{~h}$ time resolution are displayed for \pm 35 days from the zero epoch of convection onset. 

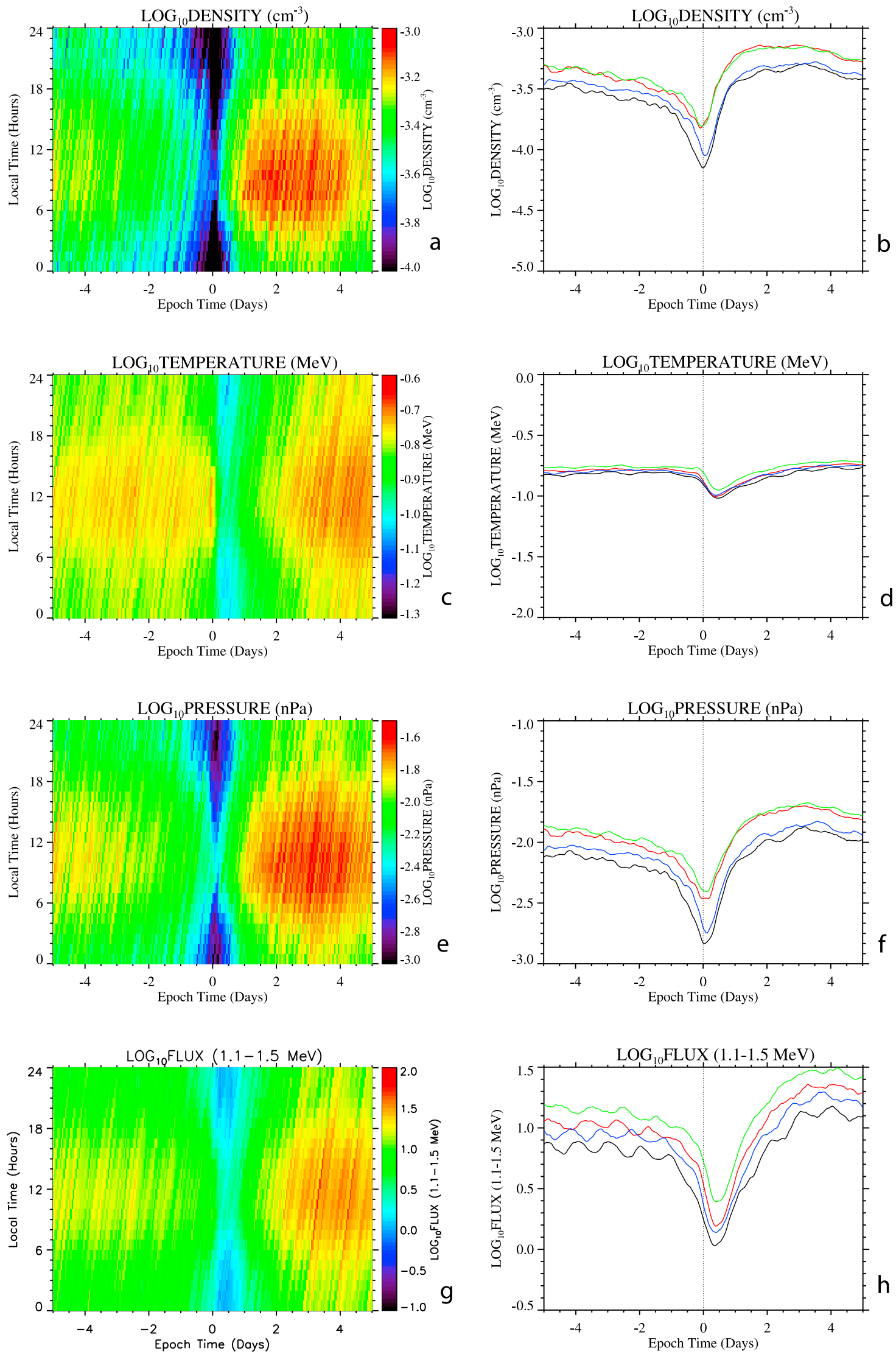

Figure 7 
Table 1. Overview of the Evolution of the Outer Electron Radiation Belt at Geosynchronous Orbit During a High-Speed-Stream-Driven Storm

\begin{tabular}{|c|c|c|c|c|c|}
\hline Phase of Storm & Number Density (n) & Temperature $(\mathrm{T})$ & Pressure $(\mathrm{P})$ & Relativistic Flux (F) & Flux Responds to \\
\hline $\begin{array}{l}\text { Calm before the storm } \\
\text { Relativistic-electron dropout } \\
\text { at storm onset }\end{array}$ & $\begin{array}{l}\text { decreases slowly } \\
\text { decreases suddenly }\end{array}$ & $\begin{array}{c}\text { constant } \\
\text { apparent decrease } \\
\text { (see section 3) }\end{array}$ & $\begin{array}{c}\text { decreases slowly } \\
\text { decreases suddenly }\end{array}$ & $\begin{array}{c}\text { decreases slowly } \\
\text { decreases suddenly }\end{array}$ & $\begin{array}{c}\text { density } \\
\text { density and temperature }\end{array}$ \\
\hline $\begin{array}{l}\text { Recovery from } \\
\text { relativistic-electron dropout }\end{array}$ & increases suddenly & $\begin{array}{l}\text { apparent increase } \\
\text { (see section 3) }\end{array}$ & increases suddenly & increases suddenly & density \\
\hline Long-duration storm & constant & increases slowly & increases slowly & increases greatly & temperature \\
\hline
\end{tabular}

the atmosphere [e.g., Rodger et al., 2008]. (Note that for certain wave-particle interactions such as EMIC waves, the strength of the scattering may be dependent upon the composition of the cold plasmaspheric material.) The decay rates of the outer electron radiation belt number density prior to storm onset have been quantified by Borovsky and Denton [2009b].

[18] Second, as shown in Figures $7 \mathrm{a}$ and $7 \mathrm{~b}$, around the time of storm onset the number density of the outer electron radiation belt suddenly decreases to very low levels (essentially to instrument-background levels). This is the wellknown "relativistic-electron dropout" [e.g., Freeman, 1964; Nagai, 1982; Onsager et al., 2002; Green et al., 2004; Borovsky and Denton, 2009a] usually discussed in terms of flux observations, but here also presented in terms of electron density. Coincident with the electron density dropout is an apparent decrease in the temperature of the electrons. Although background contributions to the channel counting rates were modeled explicitly in the spectral fitting procedure, the inferred temperature could be sensitive in detail to the assumed set of background rates (i.e., caution is advised with respect to interpretation of the low values of temperature). The relativistic-electron flux (Figures $7 \mathrm{~g}$ and $7 \mathrm{~h}$ ) also shows relativistic-electron dropout behavior, but the timing of the drop in number density differs slightly from the timing of the drop in flux. This is particularly noticeable in Figure 7 , where the time of minimum number density (at $\mathrm{t} \approx 0$ ) is almost half a day prior to the time of minimum flux. The occurrence (or not) of these relativisticelectron dropouts has been shown to be associated with the occurrence (or not) of a superdense plasma sheet in the magnetosphere during the storm [Denton and Borovsky, 2008, 2009]. The superdense plasma sheet has its origin in the compressed (or not) solar wind in the CIR upstream of the high-speed stream [Denton and Borovsky, 2009].

[19] Third, less than one day after the relativistic-electron dropout, the number density of the outer electron radiation belt at geosynchronous orbit increases sharply. This is the sudden "recovery from dropout" [Borovsky and Denton, 2009a], which is clearly seen in Figures $7 \mathrm{a}$ and $7 \mathrm{~b}$. Typically, but not always, the number density is higher after the recovery than it was just prior to the dropout (of course the number density had been decreasing slowly for a few days prior to the dropout proper). Also, the superposed temperature of the electrons is lower just after recovery than it was just prior to dropout. The pressure of the outer electron radiation belt reflects these density and temperature trends. The relativistic-electron flux (Figures $7 \mathrm{~g}$ and $7 \mathrm{~h}$ ) also shows this recovery from dropout. However, the recovery is more sudden when observed in terms of number density than when it is observed in terms of flux. Note that the recovery in number density occurs earlier than does the recovery in flux. By investigating the timing of changes in the different plasma populations concerned, the recovery from dropout of the outer electron radiation belt has been associated with the termination of the superdense-plasma sheet phase of the geomagnetic storm [Borovsky and Denton, 2009a].

[20] Fourthly, Figure 7 shows that in the first few days after recovery from a relativistic-electron dropout, the temperature of the outer electron radiation belt at geosynchronous orbit slowly increases with time while the number density remains approximately constant. This is best seen in the line plots of temperature and density in Figures $7 \mathrm{~b}$ and $7 \mathrm{~d}$. The slow temperature increase occurs during the elevated $K p$ of the several-days-long geomagnetic storm (cf. Figure 5f). As can be seen in Figures $7 \mathrm{~b}, 7 \mathrm{~d}, 7 \mathrm{f}$, and $7 \mathrm{~h}$, after the rapid recovery in the number density the relativistic-electron fluxes at geosynchronous increase by around an order of magnitude during an extended interval of elevated $K p$. These trends are noted in Table 1. It is also clear from Figure 7 that this increase in fluxes by an order of magnitude occurs while the temperature only increases by a modest amount. This comparison highlights the dichotomy of a great particle accelerator versus a modest heater (as was noted by Borovsky et al. [1998]). Commensurate with this density-temperature behavior, the pressure (thermal-energy density) of the outer electron radiation belt increases steadily for days after storm onset (Figures $7 \mathrm{e}$ and $7 \mathrm{f}$ ). The heating rates of the outer electron radiation belts at geosynchronous storms (and correlated parameters) remain to be quantified.

\section{Evolution of the Outer Electron Radiation Belt During CME-Driven Storms}

[21] In contrast to recurrent high-speed-stream-driven events wherein the electron radiation belt behavior is repeatable and easier to categorize, there are significant

Figure 7. Superposed outer electron radiation belt $(\mathrm{a}, \mathrm{b})$ number density, $(\mathrm{c}, \mathrm{d})$ temperature, $(\mathrm{e}, \mathrm{f}) \mathrm{pressure}$, and $(\mathrm{g}, \mathrm{h}) \mathrm{flux}$, calculated using a superposed-epoch analysis of 124 high-speed solar wind streams between 1993 and 2006 . Averaged values of these parameters at $1 \mathrm{~h}$ time resolution are displayed for \pm 5 days from the zero epoch of convection onset. Figures $7 \mathrm{a}$, $7 \mathrm{c}, 7 \mathrm{e}$, and $7 \mathrm{~g}$ contain enlarged versions of the data from Figure 6 . In addition, the number density, temperature, pressure, and flux are averaged over $3 \mathrm{~h}$ local-time bins and plotted as a function of epoch time (2300-0100 LT (black curves), 05000700 LT (red curves), 1100-1300 LT (green curves), and 1700-1900 LT (blue curves)). 


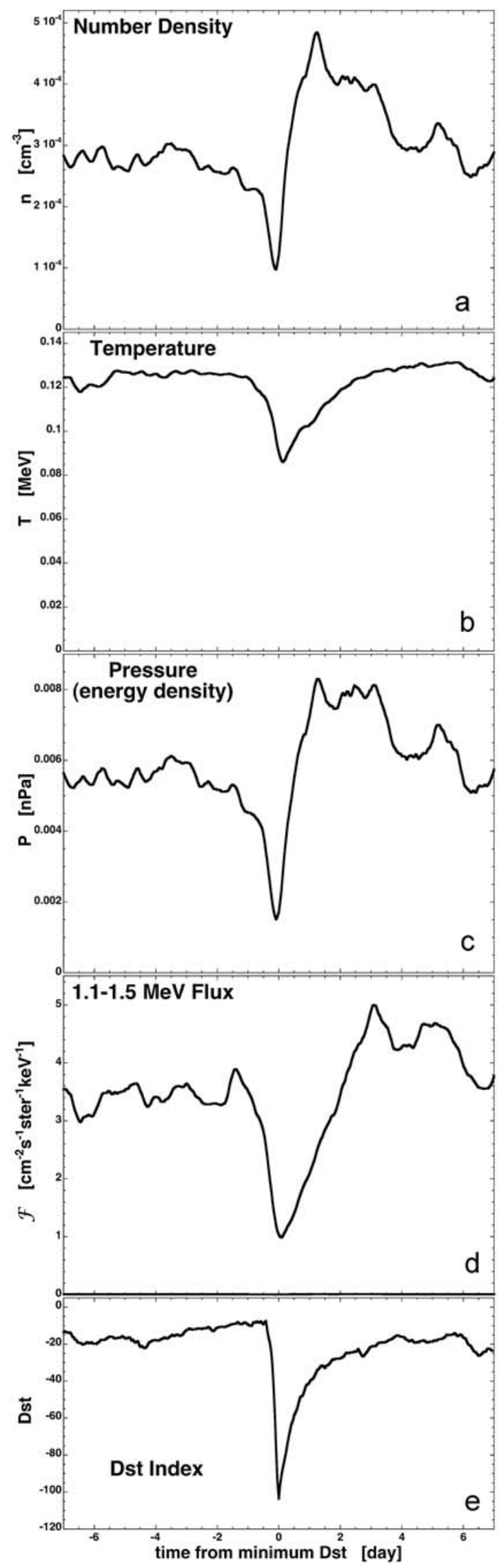

differences in solar wind drivers from one magnetic-cloud event to another [cf. Jian et al., 2006] making the electron radiation belt behavior difficult to categorize. Hence, clouddriven storms are more difficult to study via superposedepoch analysis than are high-speed-stream-driven storms. Furthermore, complications in the interpretation of the evolution of the outer electron radiation belt during CMEdriven storms arise from competing effects acting simultaneously, such as compression of the magnetosphere by solar wind ram pressure, the distortions of the inner magnetosphere by strong ring current-plasma perturbations (the Dst effect), and the evolution of the radiation belts due to geomagnetic activity. A typical (but not unique) temporal sequence for the solar wind driver is (1) interplanetaryshock arrival, (2) passage of the CME sheath, and (3) passage of the southward-IMF portion of the cloud. The shock may or may not be present, the sheath may or may not drive the magnetosphere, and the cloud may or may not have a southward-IMF portion. In addition, there may or may not be a northward-IMF portion of the cloud between the sheath and the southward-IMF portion of the cloud.

[22] For cloud-driven storms, a standard "trigger" to take for the zero epoch of the data superposition is the minimum value of the Dst index for the storm [e.g., Denton et al., 2005, 2006; Lavraud et al., 2006; Zhang et al., 2006]. Note, however, taking a single trigger at $D s t$ minimum to analyze CME-driven storms is a method that mixes several clouddriven storm phases together owing to the storm-to-storm differences in the sequences, and to the differing temporal durations of the phases. Such a single-epoch superposition of measurements from $78 \mathrm{CME}$-driven storms appears in Figure 8 , with the trigger taken to be the time of minimum Dst. The 78 storms are the CME-driven events utilized in the superposed-epoch study of magnetospheric hot plasmas by Denton et al. [2006]. In Figure 8a the superposition of the number density of the outer electron radiation belt at geosynchronous orbit is plotted, in Figure $8 \mathrm{~b}$ the superposition of the temperature is plotted, in Figure $8 \mathrm{c}$ the superposition of the pressure $\mathrm{P}=\mathrm{nk}_{\mathrm{B}} \mathrm{T}$ is plotted, in Figure $8 \mathrm{~d}$ the superposition of the $1.1-1.5 \mathrm{MeV}$ electron flux is plotted, and in Figure 8e the superposition of the $D s t$ index is plotted. The Dst plot indicates the main phase of the storm (Dst decreasing) and the recovery phase of the storm (Dst increasing back to quiet levels). As seen in Figure 8 , the general trend for the number density of the outer electron radiation belt at geosynchronous orbit is a density drop during the main phase of the storm, which commences just prior to the time of minimum Dst, and then a density increase during the recovery phase after minimum Dst. Also note that unlike the case of high-speed-stream-driven storms (e.g., Figure 7), there is only a very modest decrease of the number density of the outer electron radiation belt at

Figure 8. For 78 coronal-mass-ejection-driven storms, superpositions of the (a) number density, (b) temperature, and (c) pressure of the outer electron radiation belt at geosynchronous orbit are plotted as functions of time, as are the (d) $1.1-1.5 \mathrm{MeV}$ electron flux and (e) $D s t$ index. Each curve in Figures $8 a-8 d$ is a local-time average of the quantity. Time $\mathrm{t}=0$ is taken to be the time of minimum Dst in the storm. 
Table 2. Overview of the Evolution of the Outer Electron Radiation Belt at Geosynchronous Orbit During a Coronal Mass EjectionDriven Storm

\begin{tabular}{|c|c|c|c|c|c|c|}
\hline Zero Epoch & Phase of Storm & Number Density (n) & Temperature $(\mathrm{T})$ & Pressure $(\mathrm{P})$ & Relativistic Flux (F) & Flux Responds Primarily to \\
\hline $\begin{array}{l}\text { Sudden storm } \\
\text { commencement }\end{array}$ & $\begin{array}{l}\text { compression of } \\
\text { magnetosphere }\end{array}$ & $\begin{array}{l}\text { decreases } \\
\text { greatly }\end{array}$ & $\begin{array}{l}\text { decreases } \\
\text { slightly }\end{array}$ & $\begin{array}{c}\text { decreases } \\
\text { greatly }\end{array}$ & $\begin{array}{c}\text { decreases } \\
\text { greatly }\end{array}$ & density \\
\hline $\begin{array}{l}\text { South interplanetary magnetic } \\
\text { field (IMF) sheath }\end{array}$ & sheath driving & decreases & decreases & decreases & decreases & density and temperature \\
\hline South IMF cloud & cloud driving & increases & decreases & increases & holds steady & density and temperature \\
\hline Minimum $D s t$ & recovery & increases & increases & increases & increases & density and temperature \\
\hline
\end{tabular}

geosynchronous orbit in the several days prior to storm onset due to the lack of an extended calm before the storm. The general trend of the temperature of the outer electron radiation belt at geosynchronous orbit (Figure $8 \mathrm{~b}$ ) is a temperature decrease during the main phase and a temperature recovery during the recovery phase. The trend in the pressure of the outer electron radiation belt follows the trend in the number density: a decrease in the pressure during the main phase and an increase in the pressure during the recovery phase. The trend of the relativistic-electron flux during CME-driven storms (Figure 8d) is more like the simple trend in the outer electron radiation belt temperature than the complicated trend in the density: the flux exhibits a decrease during the main phase of the storm followed by an increase during the recovery phase. The temporal width of the decrease in the flux (Figure 8d) is much greater than the temporal width of the decrease in number density (Figure 8a). In addition, the relativistic-electron flux after the CMEdriven storms is slightly elevated initially, and then falls over the next $\sim 5$ days to a flux roughly equal to the relativisticelectron flux before the storms.

[23] In the remainder of this section, four different zero epochs (triggers) for the data superpositions are used to piecewise analyze the evolution of cloud-driven storms. All interpretation of the superposed data is restricted to the temporal vicinity of the trigger. The zero epochs used below are (1) trigger on the arrival of the interplanetary shock, (2) trigger on the beginning of sheath, (3) trigger on the beginning of southward-IMF portion of cloud, and (4) trigger on the end of southward-IMF portion of cloud. The results of this analysis are summarized in Table 2.

[24] First, the reaction of the outer electron radiation belt at geosynchronous orbit to the arrival of the interplanetary shock and compressed CME-sheath plasma is examined. In the data superposition shown in Figure 9 the zero epoch is taken to be the sudden storm commencement (SSC) at Earth, obtained from a collection of 37 very strong SSC events. The SSCs were selected for their strong compression of the magnetosphere with both a strong positive-Dst signature and a sustained sudden increase in the solar wind ram pressure (as determined from examination of the OMNI2 database). Figure 9 contains plots of the superpositions of the outer electron radiation belt number density n (Figure 9a), temperature T (Figure 9b), pressure $\mathrm{P}$ (Figure 9c), and 1.11.5 MeV electron flux (Figure 9d) at geosynchronous orbit. The ram pressure $n v^{2}$ of the solar wind is also plotted (Figure 9e): note the sudden increase at $\mathrm{T}=0$ followed by a sustained (for several hours) high pressure. The superposition of the Dst index for these events is also shown (Figure 9f). Note the sharp positive Dst deflection associated with interplanetary-shock arrival (at $\mathrm{t}=0$ ). As can also be seen, the arrival of the shock results in sudden strong decreases in the number density (Figure 9a) and the pressure (Figure 9c) of the outer electron radiation belt. The temperature of the outer electron radiation belt (Figure 9b) shows a slight decrease commencing at the time of SSC. The relativistic-electron flux F (Figure 9d) also decreases. These changes are noted in Table 2. The fractional decrease in the flux close to zero epoch is less than the fractional decrease in density: comparing the values at time $\mathrm{t}=+2 \mathrm{~h}$ with the values at $\mathrm{t}=-1 \mathrm{hr}$ the flux decreases to $58 \%$ of its initial value while the number density decreases to $\sim 42 \%$ of its initial value. The density decrease at geosynchronous orbit at time $\mathrm{t}=0$ is probably owed to a rapid compression of the magnetosphere by the increase in solar wind ram pressure (higher velocity and higher density) behind the interplanetary shock, bringing more tenuous outer magnetospheric regions to geosynchronous orbit. After the shock passage at time $t=0$, the Earth's magnetosphere is within the compressed CME-sheath plasma ahead of the coronal mass ejection. Depending on the orientation of the IMF in this sheath plasma, the sheath may or may not drive geomagnetic activity in the Earth's magnetosphere. The steady decrease in the value of Dst after zero epoch indicates that on average there is some driving by the sheath for these events.

[25] In Figure 10 a set of 16 CME-driven storm events wherein the IMF of the sheath plasma is distinctly southward is examined. Figure 10 contains plots of the superpositions of the outer electron radiation belt number density $\mathrm{n}$ (Figure 10a), temperature $\mathrm{T}$ (Figure 10b), pressure $\mathrm{P}$ (Figure 10c), and 1.1-1.5 MeV electron flux (Figure 10d) at geosynchronous orbit. The superposition of the $K p$ index for these events is also shown (Figure 10e). Time $t=0$ is taken to be the onset of the southward IMF (GSM) at Earth in the sheath plasma. The sudden increase in $K p$ near $\mathrm{t}=0$ indicates the onset of driving by the sheath plasmas; note that the $K p$ index has a $3 \mathrm{~h}$ resolution so the increase in $K p$ begins approximately $3 \mathrm{~h}$ prior to $\mathrm{t}=0$. The temporal profiles of the number density, the temperature, the pressure, and the relativistic-electron flux are all very similar to the profiles in Figure 9 where $\mathrm{t}=0$ was the SSC (arrival of the interplanetary shock). This similarity may indicate that the reaction of the outer electron radiation belt at geosynchronous orbit is dominated by the compression of the magnetosphere.

[26] To study the reaction of the outer electron radiation belt at geosynchronous orbit when the magnetosphere is driven (strongly) by the southward IMF of a magnetic cloud, a subset of 13 well-defined magnetic clouds with southward-IMF (GSM) portions was extracted from the set of Dst storms that was used in the study by Denton et al. [2005]. These magnetic clouds were identified using SWEPAM plasma measurement [McComas et al., 1998] and MAG magnetic field measurements [Smith et al., 1998] 

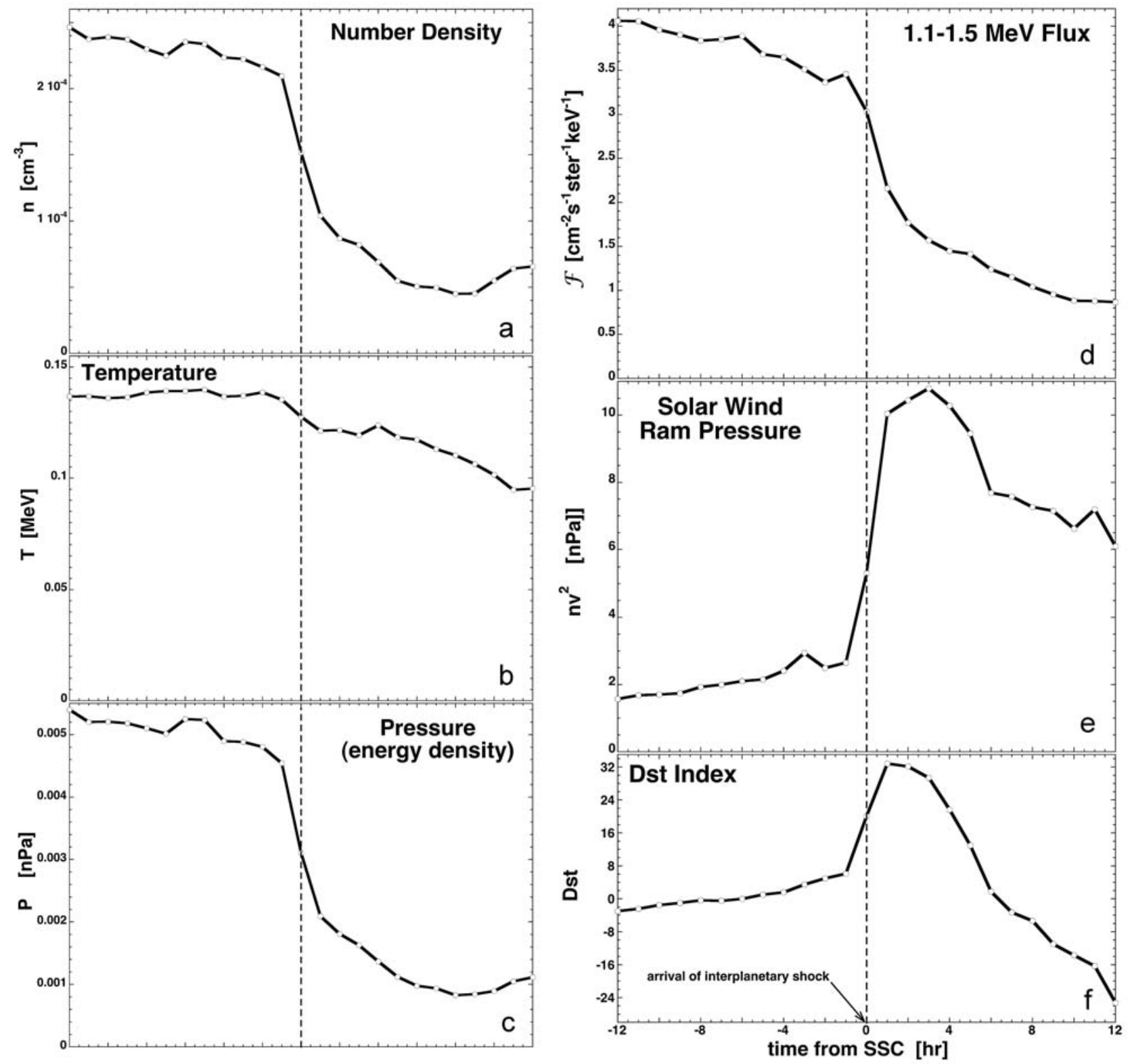

Figure 9. For 37 well-defined SSC (storm sudden commencement) events, superpositions of the (a) number density, (b) temperature, and (c) pressure of the outer electron radiation belt at geosynchronous orbit are plotted as functions of time, as are the (d) $1.1-1.5 \mathrm{MeV}$ electron flux, (e) ram pressure in the solar wind, and (f) Dst index. Each curve in Figures 9a-9d is a local-time average of the quantity. Time $\mathrm{t}=0$ is taken to be the time of SSC (arrival at Earth of an interplanetary shock).

from onboard the ACE satellite upstream of the Earth. The clouds in the solar wind were identified by their having a well-ordered magnetic field, low solar wind ion temperatures, and bidirectional electron heat flux. In Figure 11 a superposition of the measurements of the outer electron radiation belt at geosynchronous orbit is created by setting the zero epoch of the analysis to be the onset of the southward-IMF portions of those 13 magnetic clouds. Figure 11 contains plots of the superposed averages of the outer electron radiation belt number density $\mathrm{n}$ (Figure 11a), temperature T (Figure 11b), pressure P (Figure 11c), and 1.1-1.5 MeV electron flux F (Figure 11d) at geosynchronous orbit. The superposition of the $K p$ index for these events is also shown (Figure 11e). Note that immediately prior to the southward-IMF portions of clouds there can be (1) sheath plasma (which may or may not drive geomagnetic activity) or (2) northward-IMF magnetic cloud (which will not drive geomagnetic activity). As can be seen in Figure $11 \mathrm{e}, K p$ was elevated prior to $\mathrm{t}=0$ in the superposition, an indication that there was some driving prior to zero epoch in at least some of these events. As can be seen in Figures 11a and 11c, the number density and the pressure begin to increase when the southward-IMF portion of the cloud arrives. This increase with time during the southward IMF of the cloud is associated with the strong geomagnetic activity in the Earth's magnetosphere, as shown in Figure 11e. 


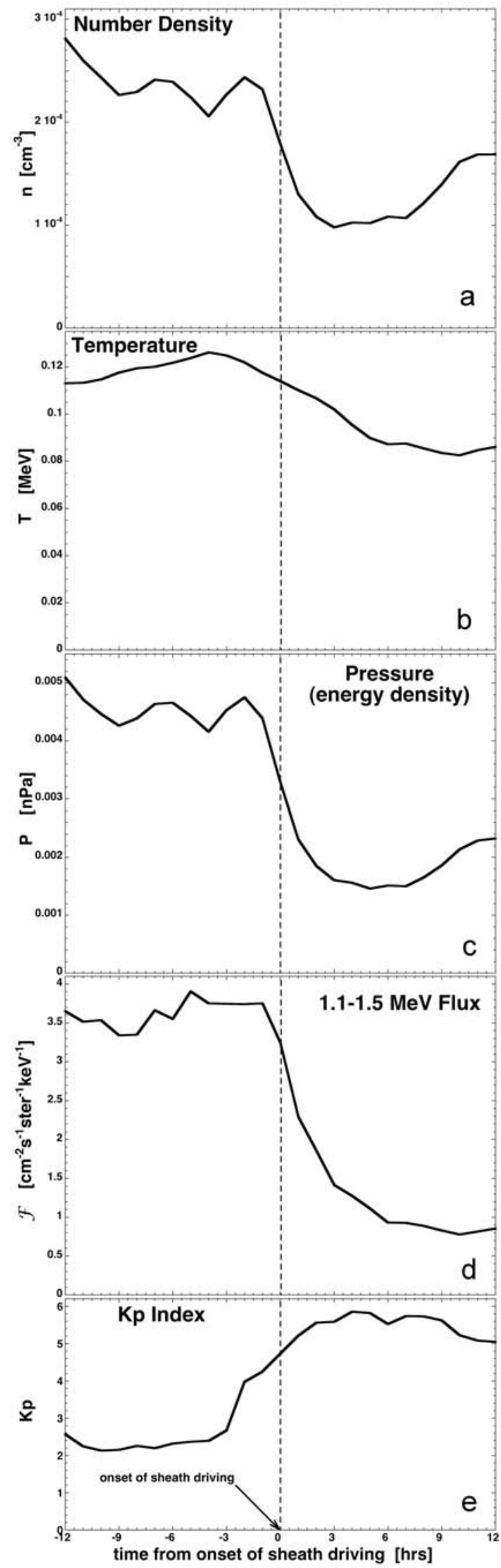

As can be seen in Figure 11b, the temperature of the outer electron radiation belt at geosynchronous orbit, which was decreasing with time before the arrival of the southwardIMF portion of the cloud, continues to decrease during the southward-IMF portion of the cloud. Note that, at time $t=0$ in Figure 11, the number density $\mathrm{n}$ of the outer electron radiation belt is quite low $\left(\sim 1 \times 10^{-4} \mathrm{~cm}^{-3}\right)$ and the temperature is also quite low $(\sim 100 \mathrm{keV})$. The temporal evolution of the relativistic-electron flux (Figure 11d) differs from the behavior of the number density and temperature. Specifically, the flux remains pretty much constant while the density increases and the temperature decreases slightly.

[27] In Figure 12 the properties of the outer electron radiation belt at geosynchronous orbit are shown with the zero epoch of the analysis set as the minimum value of $D s t$ for CME-driven storms. Seventeen cloud-driven storms with large, single-dip, negative- $D s t$ perturbations were selected from the list of Dst storms first used by Denton et al. [2006]. Triggering on the minimum value of Dst (by definition the end of the storm main phase and beginning of the storm recovery phase) likely represents the end of strong southward-IMF driving of the cloud-driven storm. Figure 12 contains plots of the superposed averages of the outer electron radiation belt number density $\mathrm{n}$ (Figure 12a), temperature $\mathrm{T}$ (Figure 12b), pressure $\mathrm{P}$ (Figure 12c), and 1.1-1.5 MeV electron flux F (Figure 12d) at geosynchronous orbit. The superposition of the Dst index for these events is also shown (Figure 12e). In Figure 12a the number density of the outer electron radiation belt is seen to increase steadily with time as Dst recovers. In Figure $12 \mathrm{~b}$ the temperature of the outer electron radiation belt at geosynchronous orbit shows a steady slow increase as Dst recovers. In Figure 12c the pressure of the outer electron radiation belt at geosynchronous orbit shows a steady increase throughout the recovery phase, tracking the evolution of the number density. The relativistic-electron flux (Figure 12d) shows the same trend as the number density and temperature: a steady increase with time as Dst recovers. These changes are noted in Table 2. The temporal profile of the relativistic-electron flux is more similar to that of the temperature than that of the number density. The evolution of the temperature and number density of the outer electron radiation belt at geosynchronous orbit around the minimum- $D$ st time may be governed by a combination of the cessation of geomagnetic activity and the inflation and deflation of the dipole magnetic field owing to the "Dst effect" [e.g., Dessler and Karplus, 1961; Kim and Chan, 1997].

\section{Summary}

[28] Analysis of almost one million individual biMaxwellian fits to relativistic-electron flux data has been

Figure 10. For 16 cloud-driven storm events with welldefined intervals of southward IMF (GSM) in the CME sheaths, superpositions of the (a) number density, (b) temperature, and (c) pressure of the outer electron radiation belt at geosynchronous orbit are plotted as functions of time, as are the (d) $1.1-1.5 \mathrm{MeV}$ electron flux and (e) $K p$ index. Each curve in Figures $10 \mathrm{a}-10 \mathrm{~d}$ is a localtime average of the quantity. Time $t=0$ is taken to be the onset of the southward-IMF interval in the sheath plasma. 


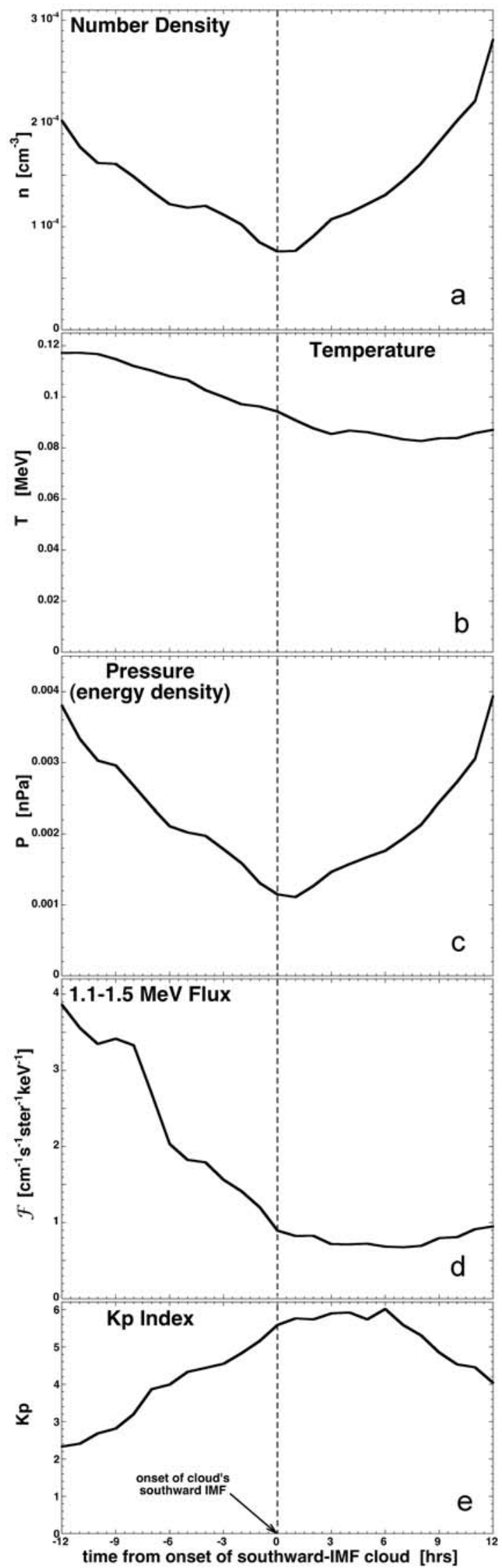

performed. The findings of the study are summarized below. These findings demonstrate the strength of a densitytemperature description of the outer electron radiation belt at geosynchronous orbit.

\subsection{General Properties of the Outer Electron Radiation Belt}

[29] Statistical analyses of the outer electron radiation belt at geosynchronous orbit are described in section 2. Salient findings are as follows.

[30] 1. At geosynchronous orbit, the median value of the number density is $\mathrm{n}=3.7 \times 10^{-4} \mathrm{~cm}^{-3}$, the median value of the temperature is $\mathrm{T}=142 \mathrm{keV}$, and the median value of the $1.1-1.5 \mathrm{MeV}$ electron flux $\mathrm{F}$ is $4.7 \mathrm{~cm}^{-2} \mathrm{~s}^{-1} \mathrm{ster}^{-1}$ $\mathrm{keV}^{-1}$. The median value of the pressure $\mathrm{P}=\mathrm{nk}_{\mathrm{B}} \mathrm{T}$ is $8.3 \times 10^{-3} \mathrm{nPa}$.

[31] 2. The fractional variations of the number density $n$ and the temperature $\mathrm{T}$ are less than the fractional variations in the relativistic-electron flux $\mathrm{F}$.

[32] 3. The density, temperature, and the relativisticelectron flux all show similar local-time dependencies, with peaks in all three quantities near local noon and minima in the quantities near local midnight. The local-time variation of the temperature is weaker than the local-time variations in $\mathrm{n}$ and $\mathrm{F}$.

[33] 4. There are systematic solar-cycle dependencies on $\mathrm{n}$, $\mathrm{T}$, and $\mathrm{F}$. The number density $\mathrm{n}$ and temperature $\mathrm{T}$ are higher during the declining phase than during the other phases of the solar cycle. The relativistic-electron flux $\mathrm{F}$ reflects the number-density and temperature trends.

\subsection{Evolution During High-Speed-Stream-Driven Storms}

[34] In section 3, superposed-epoch analysis was used to study the evolution of the outer electron radiation belt at geosynchronous orbit during high-speed-stream-driven storms. The findings of that investigation are as follows.

[35] 1. Associated with high-speed-stream-driven storms, there are clear 27 day periodicities in the number density n, temperature $\mathrm{T}$, and relativistic-electron flux $\mathrm{F}$, of the outer electron radiation belt at geosynchronous orbit.

[36] 2. For several days prior to high-speed-stream-driven storms, the number density $\mathrm{n}$ of the outer electron radiation belt decreases slowly while the temperature $\mathrm{T}$ remains approximately constant. The relativistic-electron flux $\mathrm{F}$ also decreases slowly during this period. The decrease in $\mathrm{n}$ is attributable to the build up of the outer plasmasphere, and associated pitch angle scattering during calms before storms. The decrease in $\mathrm{F}$ is attributable to the decrease in $\mathrm{n}$.

[37] 3. Near the onset time of a high-speed-stream-driven storm there is a sudden drop in the number density $\mathrm{n}$ of the outer electron radiation belt at geosynchronous orbit. The

Figure 11. For 13 cloud-driven storm events with welldefined southward-IMF (GSM) portions, superpositions of the (a) number density, (b) temperature, and (c) pressure of the outer electron radiation belt at geosynchronous orbit are plotted as functions of time, as are the (d) $1.1-1.5 \mathrm{MeV}$ electron flux and (e) $K p$ index. Time $\mathrm{t}=0$ is taken to be the onset of the southward-IMF portions of the clouds. 


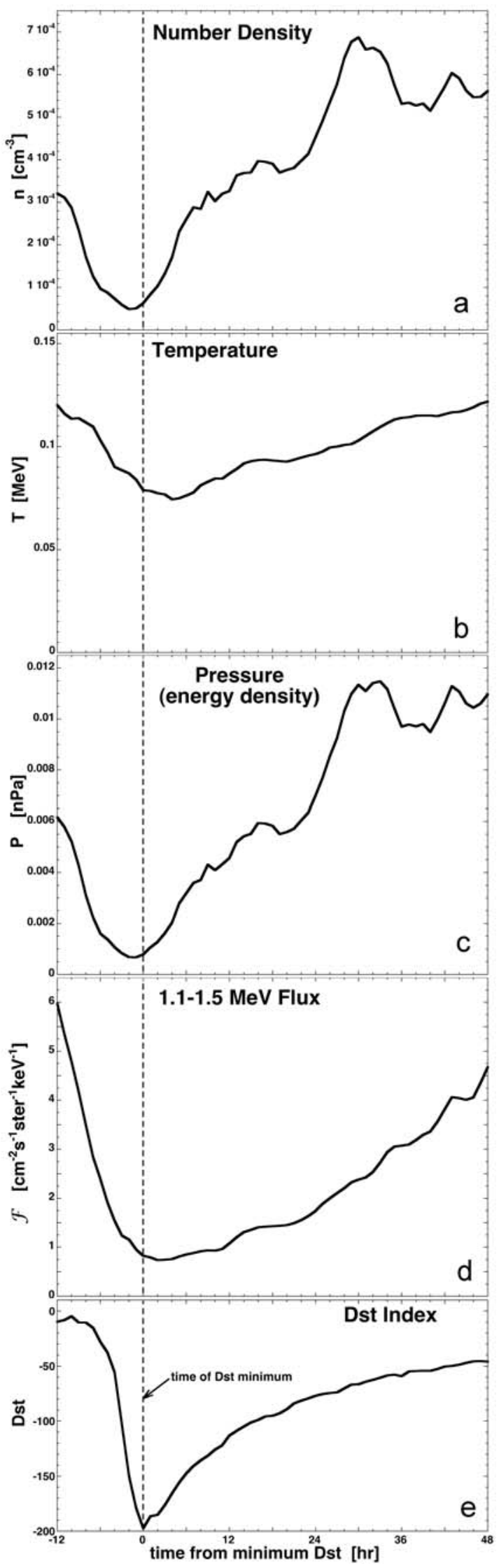

relativistic-electron flux $\mathrm{F}$ also decreases suddenly, but the flux drop is later in time than the number-density drop. This sudden decrease is the well known "relativistic-electron dropout" of high-speed-stream-driven storms.

[38] 4. Less than one day after its sudden drop, the number density $\mathrm{n}$ of the outer electron radiation belt shows a sudden increase (the "recovery from dropout"). The relativistic-electron flux $\mathrm{F}$ also shows this recovery from dropout, but the flux recovery takes longer than the numberdensity recovery.

[39] 5. After the recovery from dropout, the temperature $\mathrm{T}$ of the outer electron radiation belt at geosynchronous orbit slowly increases with time during the several days of the high-speed-stream-driven storm while the number density is approximately constant. During this time interval the relativistic-electron flux $\mathrm{F}$ steadily increases as the temperature increases at constant density. This steady increase in the relativistic-electron flux during the storm is attributable to a steady heating of the outer electron radiation belt.

[40] 6. The number density $\mathrm{n}$ of the outer electron radiation belt at geosynchronous orbit tends to be higher after HSS-driven storms than it was before such storms. The temperature $T$ of the outer electron radiation belt tends to be about the same after a storm as before. The relativisticelectron flux $\mathrm{F}$ tends to be higher after a storm than before the storm.

\subsection{Evolution During CME-Driven Storms}

[41] In section 4, superposed-epoch analysis with multiple epoch selections was used to study the evolution of the outer electron radiation belt at geosynchronous orbit during $\mathrm{CME}$ storms. The findings of that investigation are the following.

[42] 1. Unlike the case for high-speed-stream-driven storms, there is only a very modest temporal decrease in the number density $\mathrm{n}$ or relativistic-electron flux $\mathrm{F}$ of the outer electron radiation belt at geosynchronous orbit in the days prior to the onset of CME-driven storms. This is likely associated with the lack of a systematic calm before CME-driven storms.

[43] 2. When the storm sudden commencement (SSC) occurs (arrival of interplanetary shock at Earth), the number density $\mathrm{n}$ and the relativistic-electron flux $\mathrm{F}$ of the outer electron radiation belt at geosynchronous orbit both decrease suddenly. The fractional decrease in the number density is larger than the fractional decrease in the relativistic-electron flux. The temperature decrease at the time of SSC is very slight. The sudden density decrease is attributable to a sudden compression of the magnetosphere delivering higher-L energetic electrons to geosynchronous orbit.

[44] 3. During the southward-IMF (GSM) portions of magnetic clouds (storm main phase), the number density $n$ of the outer electron radiation belt at geosynchronous orbit

Figure 12. For 17 magnetic-cloud-driven storms with strong, single-dip Dst perturbations, superpositions of the (a) number density, (b) temperature, and (c) pressure of the outer electron radiation belt at geosynchronous orbit are plotted as functions of time, as are the (d) $1.1-1.5 \mathrm{MeV}$ electron flux and (e) $D s t$ index. Each curve in Figures $12 \mathrm{a}-$ $12 \mathrm{~d}$ is a local-time average of the quantity. Time $\mathrm{t}=0$ is taken to be the time of minimum Dst. 
increases with time while the temperature $\mathrm{T}$ decreases with time. The relativistic-electron flux $F$ remains approximately constant during the southward-IMF portions of the magnetic clouds.

[45] 4. During the recovery phase of CME-driven storms (the interval wherein Dst increases toward quiet time values after the time of minimum $D s t$ ) the number density n of the outer electron radiation belt at geosynchronous orbit increases and the temperature $\mathrm{T}$ increases. During the recovery phase the relativistic-electron flux $\mathrm{F}$ also increases, mimicking the temporal profile of the temperature more than the temporal profile of the number density.

[46] 5. The number density $n$ and relativistic-electron flux $\mathrm{F}$ of the outer electron radiation belt at geosynchronous orbit have slightly elevated values after a CME-driven storm as they did before the storm: these decrease to prestorm values after a few days. The temperature has a very similar value after a CME-driven storm as prior to the storm.

\subsection{Differences Between a Density-Temperature Description and a Flux Description}

[47] The relativistic-electron flux is a measure of the number of particles in the tail of the energy distribution of the outer electron radiation belt. Here, specifically, the relativistic-electron flux $\mathrm{F}$ is taken to be the flux of particles in the $1.1-1.5 \mathrm{MeV}$ energy band, with the temperature being on the order of $150 \mathrm{keV}$. One could consider the relativistic-electron flux $\mathrm{F}$ to be controlled by a combination of $\mathrm{n}$ and $\mathrm{T}$, with the flux increasing as $\mathrm{n}$ increases or as $\mathrm{T}$ increases. Through the different phases of geomagnetic storms, the number density $\mathrm{n}$, the temperature $\mathrm{T}$, and the relativistic-electron flux $\mathrm{F}$ of the outer electron radiation belt at geosynchronous orbit all show different evolutionary trends. In general the fractional variations in the relativisticelectron flux $\mathrm{F}$ are larger than the fractional variations in $\mathrm{n}$ or $\mathrm{T}$, but during some phases of storms the fractional variations in $\mathrm{F}$ can be smaller.

[48] Sometimes the relativistic-electron flux F reacts more to changes in the temperature $\mathrm{T}$ of the outer electron radiation belt than to changes in the number density $n$. One important example of this is the steady increase in the relativistic-electron flux as the outer electron radiation belt is slowly heated during several-days-long high-speedstream-driven storms.

[49] Sometimes the relativistic-electron flux F reacts more to changes in the number density $\mathrm{n}$ of the outer electron radiation belt than to changes in the temperature $T$. One example of this is the several-days-long slow decay of both $\mathrm{n}$ and $\mathrm{F}$ during calms before high-speed-stream-driven storms. A second example of this is the strong drop of both $\mathrm{n}$ and $\mathrm{F}$ during SSC compressions of the magnetosphere before CME-driven storms.

[50] Sometimes the relativistic-electron flux $F$ reacts to changes in both $\mathrm{n}$ and $\mathrm{T}$. One example of this is the relativistic-electron flux $\mathrm{F}$ remaining constant while the number density $\mathrm{n}$ increases and the temperature $\mathrm{T}$ decreases during the southward-IMF portions of magnetic clouds. A second example is the relativistic-electron flux $\mathrm{F}$ increasing while $\mathrm{n}$ and $\mathrm{T}$ both increase during the recovery phase of CME-driven storms.

[51] There are timing differences between the density and temperature $\mathrm{n}$ and $\mathrm{T}$ and the relativistic-electron flux F. One example of this is the relativistic-electron dropout, which occurs first in the number density $\mathrm{n}$ and then later in the relativistic-electron flux F. Likewise, the recovery from dropout occurs first in the number density and then later in the relativistic-electron flux.

\section{Future Work}

[52] In this report we have statistically analyzed the densities and temperatures of the outer electron radiation belt at geosynchronous orbit and have described the stormtime evolution of the outer electron radiation belt in terms of densities and temperatures. We think that this densitytemperature description removes some of the uncertainties that a flux-only description contains, since changes in the flux can be caused by changes in the number of electrons and/or by changes in the temperature of the electrons. The density-temperature description also provides a simple means to calculate the specific entropy (density of adiabatic invariants per unit flux) of the outer electron radiation belt, which can provide information about nature of changes to the radiation belts. The density-temperature description offers a simple method to quantify electron-loss rates and to quantify number fluxes and energy fluxes into the atmosphere [cf. Borovsky and Denton, 2009b]. The densitytemperature description also offers a simple method to correlate increases in the electron content of the outer electron radiation belt with the densities of potential source populations in the magnetosphere and in the solar wind. In addition, the density-temperature description provides a simple method to look at electron energization mechanisms and to quantify energy budgets.

[53] Acknowledgments. The authors would like to thank the Space Physics Data Facility (SPDF) and National Space Science Data Center (NSSDC) for providing the OMNI2 database, the World Data Centre C1 at Rutherford-Appleton Laboratory in the UK for provision of the geomagnetic indices used in this study, and Bob McPherron for providing an updated list of stream interfaces. We also thank the U.S. Air Force Research Laboratory, Hanscom Air Force Base, Massachusetts, for providing the Midnight Boundary Index. We thank Reiner Friedel for graciously running the relativistic fits to the original SOPA data, and we thank Reiner Friedel and Michelle Thomsen for helpful conversations. This work was supported by the NASA Living With a Star TR\&T Program and by the Los Alamos National Laboratory LDRD Program. Parts of this work were carried out at Los Alamos National Laboratory during summer 2008, and M.H.D. wishes to thank ISR-1 and particularly J.E.B. for financial support and hospitality during this visit.

[54] Amitava Bhattacharjee thanks Viviane Pierrard for her assistance in evaluating this paper.

\section{References}

Baker, D. N., and S. G. Kanekal (2008), Solar cycle changes, geomagnetic variations, and energetic particle properties in the inner magnetosphere, J. Atmos. Sol. Terr. Phys., 70, 195-206, doi:10.1016/j.jastp.2007.08.031.

Belian, R. D., G. R. Gisler, T. Cayton, and R. Christensen (1992), High-Z energetic particles at geostationary orbit during the great proton event of October 1989, J. Geophys. Res., 97, 16,897-16,906, doi:10.1029/ 92JA01139.

Birn, J., M. F. Thomsen, J. E. Borovsky, G. D. Reeves, D. J. McComas, R. D. Belian, and M. Hesse (1998), Substorm electron injections: Geosynchronous observations and test particle simulations, J. Geophys. Res., 103, 9235-9248, doi:10.1029/97JA02635

Borovsky, J. E., and M. H. Denton (2006a), Differences between CMEdriven storms and CIR-driven storms, J. Geophys. Res., 111, A07S08, doi:10.1029/2005JA011447.

Borovsky, J. E., and M. H. Denton (2006b), Effect of plasmaspheric drainage plumes on solar-wind/magnetosphere coupling, Geophys. Res. Lett., 33, L20101, doi:10.1029/2006GL026519. 
Borovsky, J. E., and M. H. Denton (2008), A statistical look at plasmaspheric drainage plumes, J. Geophys. Res., 113, A09221, doi:10.1029/ 2007JA012994.

Borovsky, J. E., and M. H. Denton (2009a), Relativistic-electron dropouts and recovery: A superposed epoch study of the magnetosphere and the solar wind, J. Geophys. Res., 114, A02201, doi:10.1029/2008JA013128.

Borovsky, J. E., and M. H. Denton (2009b), Electron loss rates from the outer radiation belt caused by filling of the outer plasmasphere: The calm before the storm, J. Geophys. Res., 114, A11203, doi:10.1029/2009JA014063.

Borovsky, J. E., and J. T. Steinberg (2006), The "calm before the storm" in $\mathrm{CIR} /$ magnetosphere interactions: Occurrence statistics, solar wind statistics, and magnetospheric preconditioning, J. Geophys. Res., 111, A07S10, doi:10.1029/2005JA011397.

Borovsky, J. E., M. F. Thomsen, and D. J. McComas (1997), The superdense plasma sheet: Plasmaspheric origin, solar wind origin, or ionospheric origin?, J. Geophys. Res., 102, 22,089-22,097.

Borovsky, J. E., M. F. Thomsen, D. J. McComas, T. E. Cayton, and D. J. Knipp (1998), Magnetospheric dynamics and mass flow during the November 1993 storm, J. Geophys. Res., 103, 26,373-26,394, doi:10.1029/97JA03051.

Callis, L. B., D. N. Baker, J. B. Blake, J. D. Lambeth, R. E. Boughner, M. Natarajan, R. W. Klebesadel, and D. J. Gorney (1991), Precipitating relativistic electrons: Their long-term effect on stratospheric odd nitrogen levels, J. Geophys. Res., 96, 2939-2976, doi:10.1029/90JD02184

Cayton, T. E., and R. D. Belian (2007), Numerical modeling of the synchronous orbit particle analyser (SOPA, Version 2) that flew on S/C 1990-095, LA Rep. LA-14335, Los Alamos Natl. Lab., Los Alamos, N. M.

Cayton, T. E., R. D. Belian, S. P. Gary, T. A. Fritz, and D. N. Baker (1989), Energetic electron components at geosynchronous orbit, Geophys. Res. Lett., 16(2), 147-150, doi:10.1029/GL016i002p00147.

Chen, Y., R. W. Friedel, G. D. Reeves, T. G. Onsager, and M. F. Thomsen (2005), Multisatellite determination of the relativistic electron phase space density at geosynchronous orbit: Methodology and results during geomagnetically quiet times, J. Geophys. Res., 110, A10210, doi:10.1029/2004JA010895.

Clilverd, M. A., T. D. G. Clark, A. J. Smith, and N. R. Thomson (1993) Observation of a decrease in mid-latitude whistler mode signal occurrence prior to geomagnetic storms, J. Atmos. Terr. Phys., 55, 1479-1485, doi:10.1016/0021-9169(93)90113-D.

Denton, M. H., and J. E. Borovsky (2008), Superposed epoch analysis of high-speed-stream effects at geosynchronous orbit: Hot plasma, cold plasma, and the solar wind, J. Geophys. Res., 113, A07216, doi:10.1029/2007JA012998.

Denton, M. H., and J. E. Borovsky (2009), The superdense plasma sheet in the magnetosphere during high-speed-stream-driven storms: Plasma transport and timescales, J. Atmos. Sol. Terr. Phys., 71, 1045-1058, doi:10.1016/j.jastp.2008.04.023

Denton, M. H., M. F. Thomsen, H. Korth, S. Lynch, J. C. Zhang, and M. W. Liemohn (2005), Bulk plasma properties at geosynchronous orbit, J. Geophys. Res., 110, A07223, doi:10.1029/2004JA010861.

Denton, M. H., J. E. Borovsky, R. M. Skoug, M. F. Thomsen, B. Lavraud, M. G. Henderson, R. L. McPherron, J. C. Zhang, and M. W. Liemohn (2006), Geomagnetic storms driven by ICME- and CIR-dominated solar wind, J. Geophys. Res., 111, A07S07, doi:10.1029/2005JA011436.

Denton, M. H., J. E. Borovsky, R. B. Horne, R. L. McPherron, S. K Morley, and B. T. Tsurutani (2009a), Introduction to special issue on high speed solar wind streams and geospace interactions, J. Atmos. Sol. Terr. Phys., 71, 1011-1013, doi.10.1016/j.jastp.2008.09.019.

Denton, M. H., T. Ulich, and E. Turunen (2009b), Modification of midlatitude ionospheric parameters in the F2 layer by persistent high-speed solar wind streams, Space Weather, 7, S04006, doi:10.1029/ 2008SW000443.

Dessler, A. J., and R. Karplus (1961), Some effects of diamagnetic ring currents on Van Allen radiation, J. Geophys. Res., 66, 2289-2295, doi:10.1029/JZ066i008p02289.

Elphic, R. C., M. F. Thomsen, J. E. Borovsky, and D. J. McComas (1999) Inner edge of the electron plasma sheet: Empirical models of boundary location, J. Geophys. Res., 104, 22,679-22,693, doi:10.1029/ 1999JA900213.

Freeman, J. W. (1964), The morphology of the electron distribution in the outer radiation zone and near the magnetospheric boundary as observed by Explorer 12, J. Geophys. Res., 69, 1691-1723, doi:10.1029/ JZ069i009p01691.

Friedel, R. H. W., G. D. Reeves, and T. Obara (2002), Relativistic electron dynamics in the inner magnetosphere: A review, J. Atmos. Sol. Terr. Phys., 64, 265-282, doi:10.1016/S1364-6826(01)00088-8.

Georgieva, K., B. Kirov, and E. Gavruseva (2006), Geoeffectiveness of different solar drivers, and long-term variations of the correlation between sunspot and geomagnetic activity, Phys. Chem. Earth, 31, 81-87.
Green, J. C., and M. G. Kivelson (2004), Relativistic electrons in the outer radiation belt: Differentiating between acceleration mechanisms, J. Geophys. Res., 109, A03213, doi:10.1029/2003JA010153.

Green, J. C., T. G. Onsager, T. P. O'Brien, and D. N. Baker (2004), Testing loss mechanisms capable of rapidly depleting relativistic electron flux in the Earth's outer radiation belt, J. Geophys. Res., 109, A12211, doi:10.1029/2004JA010579.

Hudson, M. K., B. T. Kress, H.-R. Mueller, J. A. Zastrow, and J. B. Blake (2008), Relationship of the Van Allen radiation belts to solar wind drivers, J. Atmos. Sol. Terr. Phys., 70, 708-729, doi:10.1016/ j.jastp.2007.11.003.

Jian, L., C. T. Russell, J. G. Luhmann, and R. M. Skoug (2006), Properties of interplanetary coronal mass ejections at one AU during 1995-2004, Sol. Phys., 239, 393-436, doi:10.1007/s11207-006-0133-2.

Kavanagh, A., and M. H. Denton (2007), High-speed solar wind streams and geospace interactions, Astron. Geophys., 48, 6.24-6.26.

Kim, H.-J., and A. A. Chan (1997), Fully adiabatic changes in storm time relativistic electron fluxes, J. Geophys. Res., 102, 22,107-22,116, doi:10.1029/97JA01814.

King, J. H., and N. E. Papitashvili (2005), Solar wind spatial scales in and comparisons of hourly Wind and ACE plasma and magnetic field data, J. Geophys. Res., 110, A02104, doi:10.1029/2004JA010649.

Lavraud, B., M. F. Thomsen, J. E. Borovsky, M. H. Denton, and T. I. Pulkkinen (2006), Magnetosphere preconditioning under northward IMF: Evidence from the study of coronal mass ejection and corotating interaction region geoeffectiveness, J. Geophys. Res., 111, A09208, doi:10.1029/2005JA011566.

Li, X., D. N. Baker, M. Temerin, G. Reeves, R. Friedel, and C. Shen (2005), Energetic electrons, $50 \mathrm{keV}$ to $6 \mathrm{MeV}$, at geosynchronous orbit: Their responses to solar wind variations, Space Weather, 3, S04001, doi: 10.1029/2004SW000105.

Liemohn, M. W., and A. A. Chan (2007), Unravelling the causes of radiation belt enhancements, Eos Trans. AGU, 88(42), doi:10.1029/ 2007EO420001

Lindsay, G. M., C. T. Russell, and J. G. Luhmann (1995), Coronal mass ejections and stream interaction region characteristics and their potential geomagnetic consequences, J. Geophys. Res., 100, 16,999-17,013, doi:10.1029/95JA00525.

Longden, N., M. H. Denton, and F. Honary (2008), Particle precipitation during ICME-driven and CIR-driven geomagnetic storms, J. Geophys. Res., 113, A06205, doi:10.1029/2007JA012752.

Madden, D., and M. S. Gussenhoven (1990), Auroral Boundary Index from 1983 to 1990 , Tech. Rep. GL-TR-90-0358, Air Force Geophys. Lab. Hanscom AFB, Mass., 21 Dec.

Mann, I. R., T. P. O’Brien, and D. K. Milling (2004), Correlations between ULF wave power, solar wind speed, and relativistic electron flux in the magnetosphere: Solar cycle dependence, J. Atmos. Sol. Terr. Phys., 66 , 187-198, doi:10.1016/j.jastp.2003.10.002.

McComas, D. J., et al. (1998), An unusual coronal mass ejection: First Solar Wind Electron, Proton, Alpha Monitor (SWEPAM) results from the Advanced Composition Explorer, Geophys. Res. Lett., 25(23), 4289-4292, doi:10.1029/1998GL900174.

McPherron, R. L., and J. Weygand (2006), The solar wind and geomagnetic activity as a function of time relative to corotating interaction regions, in Recurrent Magnetic Storms: Corotating Solar Wind Streams, Geophys. Monogr. Ser., vol. 167, edited by B. T. Tsurutani et al., pp. 125-137, AGU, Washington, D. C.

McPherron, R. L., D. N. Baker, and N. U. Crooker (2009), Role of the Russell-McPherron effect in the acceleration of relativistic electrons, J. Atmos. Sol. Terr. Phys., 71, 1032-1044, doi:10.1016/j.jastp.2008.11.002.

Meredith, N. P., R. B. Horne, R. M. Thorne, D. Summers, and R. A. Anderson (2004), Substorm dependence of plasmaspheric hiss, J. Geophys. Res., 109, A06209, doi:10.1029/2004JA010387.

Millan, R. M., and R. M. Thorne (2007), Review of radiation belt relativistic electron losses, J. Atmos. Sol. Terr. Phys., 69, 362-377, doi:10.1016/j.jastp.2006.06.019.

Nagai, T. (1982), Local time dependence of electron flux changes during substorms derived from multi-satellite observations at geosynchronous orbit, J. Geophys. Res., 87, 3456-3468, doi:10.1029/JA087iA05p03456.

O'Brien, T. P., R. L. McPherron, D. Sornette, G. D. Reeves, R. Friedel, and H. J. Singer (2001), Which magnetic storms produce relativistic electrons at geosynchronous orbit, J. Geophys. Res., 106, 15,533-15,544, doi:10.1029/2001JA000052.

Onsager, T. G., G. Rostoker, H.-J. Kim, G. D. Reeves, T. Obara, H. J. Singer, and C. Smithtro (2002), Radiation belt electron flux dropouts: Local time, radial, and particle-energy dependence, J. Geophys. Res., 107(A11), 1382, doi:10.1029/2001JA000187.

Onsager, T. G., A. A. Chan, Y. Fei, S. R. Elkington, J. C. Green, and H. J. Singer (2004), The radial gradient of relativistic electrons at geosynchronous orbit, J. Geophys. Res., 109, A05221, doi:10.1029/2003JA010368. 
Onsager, T. G., J. C. Green, G. D. Reeves, and H. J. Singer (2007), Solar wind and magnetospheric conditions leading to the abrupt loss of outer radiation belt electrons, J. Geophys. Res., 112, A01202, doi:10.1029/ 2006JA011708.

Paulikas, G. A., and J. B. Blake (1979), Effects of the solar wind on magnetospheric dynamics: Energetic electrons at the synchronous orbit, in Quantitative Modeling of Magnetospheric Processes, Geophys. Monogr. Ser., vol. 21, edited by W. P. Olsen, p. 180, AGU, Washington, D. C.

Pesnell, W. D., R. A. Goldberg, C. H. Jackman, D. L. Chenette, and E. E. Gaines (2000), Variation of mesospheric ozone during the highly relativistic electron event in May 1992 as measured by the High Resolution Doppler Imager instrument on UARS, J. Geophys. Res., 105, 22,94322,953, doi:10.1029/2000JA000091.

Pierrard, V., and J. Lemaire (1996), Fitting the AE-8 energy spectra with two Maxwellian functions, Radiat. Meas., 26, 333-337, doi:10.1016/ 1350-4487(96)00057-1.

Reeves, G. D., K. L. McAdams, R. H. W. Friedel, and T. P. O’Brien (2003), Acceleration and loss of relativistic electrons during geomagnetic storms, Geophys. Res. Lett., 30(10), 1529, doi:10.1029/2002GL016513.

Rodger, C. J., T. Raita, M. A. Clilverd, A. Seppälä, S. Dietrich, N. R. Thomsen, and T. Ulich (2008), Observations of relativistic electron precipitation from the radiation belts driven by EMIC waves, Geophys. Res. Lett., 35, L16106, doi:10.1029/2008GL034804.

Roederer, J. G. (1967), On the adiabatic motion of energetic particles in a model magnetosphere, J. Geophys. Res., 72, 981-992, doi:10.1029/ JZ072i003p00981.

Roldugin, V. C., M. I. Beloglazov, and G. F. Rements (2000), Total ozone decrease in the Arctic after REP events, Ann. Geophys., 18, 332-336, doi:10.1007/s00585-000-0332-3.

Rufenach, C. L., R. F. Martin, and H. H. Sauer (1989), A study of geosynchronous magnetopause crossings, J. Geophys. Res., 94, 15,125-15,134, doi:10.1029/JA094iA11p15125.

Russell, C. T., and R. L. McPherron (1973), Semiannual variation of geomagnetic activity, J. Geophys. Res., 78, 92-108, doi:10.1029/ JA078i001p00092.

Sandanger, M. I. J., F. Søraas, M. Sørbø, K. Aarsnes, K. Oksavik, and D. S. Evans (2009), Relativistic electron losses related to EMIC waves during CIR and CME storms, J. Atmos. Sol. Terr. Phys., 71, 1126-1144, doi:10.1016/j.jastp.2008.07.006

Seppälä, A., M. A. Cliverd, and C. J. Rodger (2007), NOx enhancements in the middle atmosphere during 2003-2004 polar winter: Relative significance of solar proton events and the aurora as a source, J. Geophys. Res. 112, D23303, doi:10.1029/2006JD008326.

Sinnhuber, B. M., P. von der Gathen, M. Sinnhuber, M. Rex, G. Konig-Langlo, and S. J. Oltmans (2006), Large decadal scale changes of polar ozone suggest solar influence, Atmos. Chem. Phys., 6, 18351841 .
Smith, C. W., M. H. Acuña, L. F. Burlaga, J. L'Heureux, N. F. Ness, and J. Scheifele (1998), The ACE Magnetic Fields Experiment, Space Sci. Rev., 86, 613-632.

Sojka, J. J., R. L. McPherron, A. P. van Eyken, M. Nicolls, C. Heinselman, and J. Kelly (2009), Observations of ionospheric heating during the passage of solar coronal hole fast streams, Geophys. Res. Lett., 36, L19105, doi:10.1029/2009GL039064.

Summers, D., N. Binbin, and N. P. Meredith (2007a), Timescales for radiation belt electron acceleration and loss due to resonant wave-particle interactions: 1. Theory, J. Geophys. Res., 112, A04206, doi:10.1029/ 2006JA011801.

Summers, D., N. Binbin, and N. P. Meredith (2007b), Timescales for radiation belt electron acceleration and loss due to resonant wave-particle interactions: 2. Evaluation for VLF chorus, ELF hiss, and electromagnetic ion cyclotron waves, J. Geophys. Res., 112, A04207, doi:10.1029/ 2006JA011993.

Thomsen, M. F. (2004), Why $K p$ is such a good measure of magnetospheric convection, Space Weather, 2, S11004, doi:10.1029/2004SW000089.

Tsurutani, B. T., et al. (2006), Corotating solar wind streams and recurrent geomagnetic activity: A review, J. Geophys. Res., 111, A07S01, doi:10.1029/2005JA011273.

Turner, N. E., E. J. Mitchell, D. J. Knipp, and B. A. Emery (2006), Energetics of magnetic storms driven by corotating interaction regions: A study of geoeffectiveness, in Recurrent Magnetic Storms: Corotating Solar Wind Streams, Geophys. Monogr. Ser, vol. 167, edited by B. T. Tsurutani et al., pp. 113-124, AGU, Washington, D. C.

Wilken, B., Q.-G. Zong, G. D. Reeves, T. Doke, and T. Yamamoto (1999), Geoactivity in response to CIR/CME events: A synoptic view, Phys. Chem. Earth, 24, 113-117.

Wrenn, G. L. (2009), Chronology of 'killer' electrons: Solar cycles 22 and 23, J. Atmos. Sol. Terr. Phys., 71, 1210-1218.

Zaharia, S., M. F. Thomsen, J. Birn, and M. H. Denton (2005), Effect of storm-time plasma pressure on the magnetic field in the inner magnetosphere, Geophys. Res. Lett., 32, L03102, doi:10.1029/2004GL021491.

Zhang, J., M. W. Liemohn, M. F. Thomsen, J. U. Kozyra, M. H. Denton, and J. E. Borovsky (2006), A statistical comparison of hot-ion properties at geosynchronous orbit during intense and moderate geomagnetic storms at solar maximum and minimum, J. Geophys. Res., 111, A07206, doi:10.1029/2005JA011559.

J. E. Borovsky and T. E. Cayton, Space Science and Applications, Los Alamos National Laboratory, Los Alamos, NM 87545, USA.

M. H. Denton, Department of Communication Systems, Lancaster University, InfoLab 21, Lancaster LA1 4WA, UK. (m.denton@lancaster. ac.uk) 